\title{
Topological fluctuating electron-hole Cooper pairs in graphene-GaAs heterostructures
}

\author{
Dmitry K. Efimkin ${ }^{1,2, *}$ \\ ${ }^{1}$ School of Physics and Astronomy, Monash University, Victoria 3800, Australia \\ ${ }^{2}$ ARC Centre of Excellence in Future Low-Energy Electronics Technologies, Monash University, Victoria 3800, Australia
}

\begin{abstract}
Fluctuating Cooper pairs formed by spatially separated electrons and holes are precursors of their equilibrium condensation. Their presence strongly impacts transport phenomena and interlayer tunneling in double-layer systems above the transition temperature. Here, we consider a hybrid graphene/quantum well double-layer system and focus on the dynamics of fluctuating Cooper pairs formed by conventional electrons and Dirac holes. We demonstrate that the chiral nature of Dirac holes is manifested in the presence of two (almost) degenerate competing pairing channels, which are intertwined by effective pseudospin-orbit interactions. We argue that the spectrum of the GinzburgLandau Hamiltonian describing the energetics of fluctuating Cooper pairs is geometrically nontrivial and can be characterized by the half-integer topological Chern number. We derive a kinetic equation for fluctuating Cooper pairs and demonstrate that their nontrivial geometries generate two anomalous velocities of distinct geometrical origins. These velocities are intricately connected with the Berry curvature and the quantum metric for the Ginzburg-Landau Hamiltonian, respectively. The resulting anomalous contributions to conductivity are singular at the transition temperature, and we discuss possible setups for their experimental observation.
\end{abstract}

\section{INTRODUCTION}

Over the past decades, there has been a growing interest in the geometrical Berry phase for electrons in solids. The presence of the phase has a profound effect on material properties and is responsible for a variety of phenomena, such as polarization, orbital magnetism, and various (quantum, anomalous, or spin) Hall effects (see reviews Ref. [1-3] and references therein). Additionally, the corresponding Berry curvature, which functions as an analog of a magnetic field in reciprocal space, is a cornerstone concept for the topological classification of solids [4]. The spatial distribution of the Berry curvature in reciprocal space distinguishes topological insulators, semimetals, superconductors, and electron-hole superfluids from their topologically trivial counterparts [5-7].

Superfluids formed by closely spaced electrons and holes in bilayer systems (e.g., semiconductor GaAs/AlGaAs quantum well (QW) systems [8-13], heterostructures formed by monolayer materials [14-26], topological insulator thin films [27, 28]) are close relatives of superconductors. These superfluids can maintain phase coherence via a Cooper pair (CP) condensate and support dipolar superfluidity, which is potentially useful for various applications. Thus far, the nontrivial geometry of electron-hole superfluids has been considered only as a feature of Bogoliubov quasiparticles. The spectrum of these quasiparticles is shaped by the interplay between the band geometry of electrons and holes and the momentum distribution of the static order parameter describing the equilibrium $\mathrm{CP}$ condensate. Here, we argue that there is another geometrical aspect of electron-hole superfluids (as well as superconductors) that has been previously overlooked.

Above the transition temperature for electron-hole CP condensation, Gaussian fluctuations of the order param- eter occur, which are usually interpreted as partly coherent fluctuating CPs [29]. The presence of these fluctuations results in the strong enhancement and critical behavior of interlayer tunneling [30, 31] and the Coulomb drag resistance [32-35] reminiscent of the Aslamazov-Larkin effect and related effects in superconductors [36-40]. The behavior consistent with the presence of these fluctuations has been reported in doublebilayer graphene [41], $\mathrm{MoSe}_{2}-\mathrm{WSe}_{2}$ heterostructures [42], conventional semiconductor QWs [43-45], and hybrid graphene-GaAs bilayers [46]. Here, we demonstrate that nontrivial geometries of electrons and holes pass on to the fluctuating CPs and that their spectrum can also be classified as topological.

In the present paper, we consider the hybrid grapheneGaAs bilayer reported in Ref. [46] and sketched in Fig 1. We focus on the dynamics of fluctuating CPs formed by conventional electrons and Dirac holes. We demonstrate that the chiral nature of Dirac holes is manifested in the presence of two (almost) degenerate competing pairing channels, which are intertwined by effective pseudospinorbit interactions. We argue that the spectrum of the Ginzburg-Landau (GL) Hamiltonian for fluctuating CPs is geometrically nontrivial and can be characterized by the half-integer Chern number. We derive a kinetic equation for fluctuating CPs and demonstrate that their nontrivial geometries generate two anomalous velocities, which are intricately related to the Berry curvature and quantum metric for the GL Hamiltonian, respectively. The resulting anomalous contributions to conductivity are singular at the transition temperature, and we discuss possible setups for their experimental observation.

The remainder of the paper is organized as follows. In Section II, we present a low-energy model describing the hybrid graphene-GaAs double-layer system. In Section III, we discuss the Cooper instability and classify 

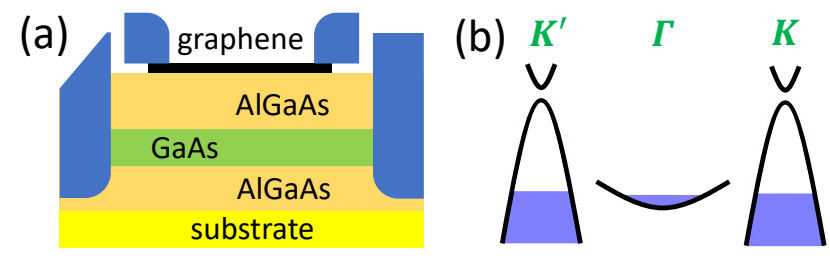

FIG. 1. Schematics of the hybrid graphene/GaAs bilayer. A graphene sheet is deposited on the surface of a semiconductor AlGaAs, underneath which a GaAs quantum well hosts two-dimensional electron gas. Carriers in the two layers are induced by gating or by a doping layer [46] (not shown in the sketch) and have independent contacts (dark blue) required for transport measurements). (b) The low-energy band structure of the hybrid graphene-GaAs bilayer includes a conduction band with conventional electrons and a pair of valence bands ( $\mathrm{K}$ and $\mathrm{K}^{\prime}$ valleys) with Dirac holes.

the pairing channels. In Section IV, we introduce the GL Hamiltonian and discuss the nontrivial geometries of its spectrum. In Section V, we derive a kinetic equation for fluctuating CPs. The temperature dependence of the anomalous paraconductivity is presented in Section VI, and different experimental setups for its detection are overviewed in Section VII. Section VIII is devoted to discussions and conclusions.

\section{MODEL}

The hybrid graphene-GaAs bilayer and its low-energy band structure are sketched in Fig. 1 (a). Following the experimental setup [46], we assume that graphene sheet with excess of holes is deposited on the surface of a semiconductor AlGaAs, underneath which a GaAs quantum well hosts two-dimensional electron gas. The physics of electron-hole bilayers formed by monolayer materials is very rich and has been considered in a number of recent papers [14-26, 47-49] (see Ref. [13] for a review) aiming to provide realistic predictions of the density/temperature phase diagram. Here, we follow a different route and consider a minimal phenomenological model that properly accounts for the chiral nature of Dirac holes.

The nature of correlations in the hybrid electron-hole bilayer depends on three dimensionless parameters. The first two are the Wigner-Seitz interaction strength parameters $r_{\mathrm{s}}^{\mathrm{e}}=m_{\mathrm{e}} e^{2} / \hbar p_{\mathrm{F}} \kappa_{\mathrm{e}}$ and $r_{\mathrm{s}}^{\mathrm{h}}=e^{2} / \hbar v \kappa_{\mathrm{h}}$ that scales the ratio of interactions and kinetic energy in GaAs QW and graphene, respectively. The third parameter $p_{\mathrm{F}} d / \hbar$ scales the interlayer Coulomb interactions respect to the intralayer one. Here $m_{\mathrm{e}}$ is mass for electron mass in GaAs QW and $p_{\mathrm{F}}$ is their Fermi momentum; $v$ is the velocity of Dirac holes in graphene; $\kappa_{\mathrm{e}}$ and $\kappa_{\mathrm{h}}$ are the effective dielectric constants for each layer in the considered multilayer heterostructure and $d$ is separation distance between electrons and holes. If $r_{\mathrm{s}}^{\mathrm{e}(\mathrm{h})} \ll 1$ and $p_{\mathrm{F}} d / \hbar \gg 1$ the system is in the weak coupling regime with pairing correlations only in the vicinity of Fermi level for electrons and holes. This regime can be described by the Bardeen-Cooper-Schrieffer (BCS) theory. Its range of the applicability in the considered hybrid bilayer also extends to the moderate coupling strength regime, $r_{\mathrm{s}}^{\mathrm{e}(\mathrm{h})} \sim 1$ and $p_{\mathrm{F}} d / \hbar \sim 1$, and is wider compared to the conventional QW bilayers. The reason is that the gapless nature of Dirac spectrum [50] does not allow the bilayer to host interlayer excitons. As a result, the strong coupling regime, $r_{\mathrm{s}}^{\mathrm{e}(\mathrm{h})} \gg 1$ and $p_{\mathrm{F}} d / \hbar \sim 1$, is not the Bose-Einstein condensate of indirect excitons, but is anticipated to be multiband BCS-like paired state [47-49] where pairing correlations also span to remote bands (empty conduction band in graphene). In reported hybrid grapheneGaAs bilayers [46] the above-listed controlling parameters can be estimated as $r_{\mathrm{s}}^{\mathrm{e}} \sim 1.1$ and $r_{\mathrm{s}}^{\mathrm{h}} \sim 0.3$ and $p_{\mathrm{F}} d / \hbar \sim 2.6$. That is why the system is in the weak-tomoderate coupling regime and can therefore be described by the phenomenological weak coupling BCS theory we develop below.

Electrons in the QW can be described by the field operator $e_{\mathbf{r}}$, and their kinetic energy is given by

$$
\hat{H}_{\mathrm{e}}=\int \mathbf{d} \mathbf{r} e_{\mathbf{r}}^{\dagger}\left(\frac{\hat{\mathbf{p}}^{2}-p_{\mathrm{F}}^{2}}{2 m_{\mathrm{e}}}\right) e_{\mathbf{r}}
$$

The spin degree of freedom is of little importance here and does not need to be treated explicitly. The lowenergy electronic states in graphene are concentrated near two inequivalent valleys ( $\mathrm{K}$ and $\mathrm{K}^{\prime}$ ), which are labeled by the index $\zeta= \pm 1$. These states are described by the spinor field operator $h_{\mathbf{r}}=\left(h_{\mathbf{r}}^{\mathrm{A}}, h_{\mathbf{r}}^{\mathrm{B}}\right)$, and their pseudospin corresponds to the sublattice (A and $\mathrm{B})$ degree of freedom of the honeycomb lattice. The kinetic energy of the Dirac states is given by

$$
H_{\mathrm{h}}=\int \mathbf{d} \mathbf{r} \hat{h}_{\mathbf{r}}^{\dagger}\left(\begin{array}{cc}
\delta+\epsilon_{\mathrm{F}}^{\mathrm{h}} & v\left(\zeta \hat{p}_{x}-i \hat{p}_{y}\right) \\
v\left(\zeta \hat{p}_{x}+i \hat{p}_{y}\right) & -\delta+\epsilon_{\mathrm{F}}^{\mathrm{h}}
\end{array}\right) \hat{h}_{\mathbf{r}} .
$$

Here $\epsilon_{\mathrm{F}}^{\mathrm{h}}$ is the Fermi energy. A small energy asymmetry between sublattices $\delta \ll \epsilon_{\mathrm{F}}^{\mathrm{h}}$ can be induced by substrate engineering. As shown in Fig. 1 (b), asymmetry opens the gap $2|\delta|$ between the partly filled valence band and the empty conduction band, which has a massive Dirac dispersion $\pm \epsilon_{\mathbf{p}}^{\mathrm{h}}$ with $\epsilon_{\mathbf{p}}^{\mathrm{h}}=\left(v^{2} \mathbf{p}^{2}+\delta^{2}\right)^{1 / 2}$. We will refer to the empty states in the valence band as holes; however, it is instructive to not perform the formal particle-hole transformation [51]. In the weak-to-moderate coupling regime, electron-hole correlations occur only within the vicinity of the Fermi level, and the empty conduction band can simply be truncated. 
The chiral nature of Dirac holes is encoded in their spinor wave function, which is given by

$$
|\mathbf{p}\rangle_{\mathrm{h}}=\left(\begin{array}{c}
\zeta \sin \left(\frac{\theta_{\mathbf{p}}}{2}\right) e^{-i \frac{\zeta \phi_{\mathbf{p}}}{2}} \\
-\cos \left(\frac{\theta_{\mathbf{p}}}{2}\right) e^{i \frac{\zeta \phi_{\mathbf{p}}}{2}}
\end{array}\right) .
$$

Here, $\phi_{\mathbf{p}}$ is the polar angle for vector $\mathbf{p}$ and $\cos \left(\theta_{\mathbf{p}}\right)=$ $\delta / \epsilon_{\mathbf{p}}^{\mathrm{h}}$. It is instructive to introduce the compact notations $c_{\mathbf{p}} \equiv \cos \left(\theta_{\boldsymbol{p}} / 2\right)$ and $s_{\mathbf{p}}=\sin \left(\theta_{\boldsymbol{p}} / 2\right)$. The important feature of Dirac holes is their geometrically nontrivial spectrum [1]. The geometry is characterized by the Berry connection $\mathbf{A}_{\mathbf{p}}^{\mathrm{h}}=i_{\mathrm{h}}\left\langle\mathbf{p}\left|\nabla_{\mathbf{p}}\right| \mathbf{p}\right\rangle_{\mathrm{h}}$, and the Berry curvature $\Omega_{\mathbf{p}}^{\mathrm{h}}=\left[\boldsymbol{\nabla}_{\mathbf{p}} \times \mathbf{A}_{\mathbf{p}}^{\mathrm{h}}\right]_{\mathbf{z}}$. The latter is valley-dependent and is given by

$$
\Omega_{\mathbf{p}}^{\mathrm{h}}=\frac{\zeta v^{2} \delta}{\left(v^{2} \mathbf{p}^{2}+\delta^{2}\right)^{3 / 2}}, \quad \mathcal{C}^{\mathrm{h}}=\int \frac{d \mathbf{p}}{2 \pi} \Omega_{\mathbf{p}}^{\mathrm{h}}=\frac{\zeta \delta}{2|\delta|} .
$$

The low-energy spectrum around each of two valleys can be characterized by the half-integer Chern number and is therefore topologically nontrivial. When the total Chern number of the two valleys is zero, the large momentum separation between the valleys provides partial topological protection and results in a number of phenomena (e.g., helical states at edges and domain walls, where the asymmetry energy $\delta$ flips its sign) known for topological insulators.

We further assume that the Fermi momentum of Dirac holes matches the momentum $p_{\mathrm{F}}$ for electrons in a QW. This is the most favorable regime for electron-hole Cooper pairing driven by an attractive Coulomb interaction. Being effectively screened by charge carriers in both layers, the attractive interactions can be approximated by the contact pseudopotential with momentumindependent Fourier transform $V$. If we neglect intervalley scattering, the interactions can be described by the following Hamiltonian:

$$
H_{\mathrm{int}}=\int d \mathbf{r} V e_{\mathbf{r}}^{\dagger} \hat{h}_{\mathbf{r}}^{\dagger} \cdot \hat{h}_{\mathbf{r}} e_{\mathbf{r}}
$$

Due to the presence of the pseudospin degree of freedom for Dirac holes, the contact interactions drives the Cooper instability in multiple pairing channels.

\section{COOPER PAIRING CHANNELS}

To analyze possible pairing channels, we assume that the $\mathrm{CP}$ condensate is at rest. The condensate can be described by the order parameter $\Delta_{\mathbf{p}}^{\mathrm{v}}=-V\left\langle h_{\mathbf{p v}}^{\dagger} e_{\mathbf{p}}\right\rangle$, where $h_{\mathbf{p v}}$ is the annihilation operator for the valence band states in graphene. Within the mean field theory, the pairing channels can be addressed with the help of the linearized self-consistent equation for the order parame- ter $\Delta_{\mathbf{p}}^{\mathrm{v}}$, which can be written as

$$
\Delta_{\mathbf{p}}^{\mathrm{v}}=V \sum_{\mathbf{p}^{\prime}} \Lambda_{\mathbf{p p}^{\prime}} \frac{\operatorname{th}\left(\frac{\xi_{\mathbf{p}^{\prime}}^{\mathrm{e}}}{2 T}\right)+\operatorname{th}\left(\frac{\xi_{\mathbf{p}^{\prime}}^{\mathrm{h}}}{2 T}\right)}{2\left(\xi_{\mathbf{p}^{\prime}}^{\mathrm{e}}+\xi_{\mathbf{p}^{\prime}}^{\mathrm{h}}\right)} \Delta_{\mathbf{p}^{\prime}}^{\mathrm{v}}
$$

Here, $\xi_{\mathbf{p}}^{\mathrm{e}(\mathrm{h})}=v_{\mathrm{e}(\mathrm{h})}\left(p-p_{\mathrm{F}}\right)$ is the dispersion of electrons (holes) linearized in the vicinity of the Fermi momentum $p_{\mathrm{F}}$. The overlap of the spinor wave functions $\Lambda_{\mathbf{p p}^{\prime}}=$ ${ }_{h}\left\langle\mathbf{p}^{\prime} \mid \mathbf{p}\right\rangle_{\mathrm{h}}$ reflects the chiral nature of Dirac holes and is given by

$$
\Lambda_{\mathbf{p p}^{\prime}}=s_{\mathbf{p}} s_{\mathbf{p}^{\prime}} e^{-i \frac{\zeta \mathbf{p}_{\mathbf{p}^{\prime}}}{2}}+c_{\mathbf{p}} c_{\mathbf{p}^{\prime}} e^{i \frac{\zeta \phi_{\mathbf{p}^{\prime}}}{2}} .
$$

Here, $\phi_{\mathbf{p} \mathbf{p}^{\prime}}$ is the angle between momenta $\mathbf{p}$ and $\mathbf{p}^{\prime}$. The presence of the factor $\Lambda_{\mathbf{p p}^{\prime}}$ reorganizes the Cooper pairing channels and plays a very important role. If we approximate $c_{\mathbf{p}}$ and $s_{\mathbf{p}}$ by their values at the Fermi level $c_{\mathrm{F}}$ and $s_{\mathrm{F}}$, the order parameter $\Delta^{\mathrm{v}}$ is momentumindependent and Eq. (6) becomes algebraic. As clearly shown, there are two competing channels with orbital quantum numbers $l= \pm 1 / 2$, and the corresponding dimensionless coupling constants are given by

$$
\begin{gathered}
\lambda_{-\frac{\zeta}{2}}=s_{\mathrm{F}}^{2} V \nu_{0}=\frac{V \nu_{0}}{2}\left(1-\frac{\delta}{\epsilon_{\mathrm{F}}^{\mathrm{h}}}\right), \\
\lambda_{\frac{\varsigma}{2}}=c_{\mathrm{F}}^{2} V \nu_{0}=\frac{V \nu_{0}}{2}\left(1+\frac{\delta}{\epsilon_{\mathrm{F}}^{\mathrm{h}}}\right) .
\end{gathered}
$$

The coupling constant $\lambda_{l}$ determines the transition temperature in the corresponding channel as

$$
T_{l}^{0}=\frac{2 \epsilon_{0} e^{\mathrm{C}}}{\pi} e^{-\frac{1}{\lambda_{l}}}
$$

Here, $C=0.577$ is the Euler constant, $\nu_{0}=p_{\mathrm{F}} / \pi \hbar^{2}\left(v_{\mathrm{e}}+\right.$ $\left.v_{\mathrm{h}}\right)$ is the effective density of states at the Fermi level, and $\epsilon_{0}=\sqrt{v_{\mathrm{e}} v_{\mathrm{h}}} p_{0}$ is determined by the momentum cutoff $p_{0}$. In the absence of sublattice asymmetry, $\delta=0$, the coupling constants match each other $\lambda_{1 / 2}=\lambda_{-1 / 2}$, and two channels are therefore degenerate (We demonstrate below that in this regime the system is unstable towards the finite momentum Cooper pairing). Due to the exponential dependence of the transition temperature on the coupling constant, the degeneracy is effectively lifted even in the presence of a very small sublattice asymmetry $\delta \ll \epsilon_{\mathrm{F}}^{\mathrm{h}}$.

The presence of two (almost) degenerate competing Cooper pairing channels is a unique feature of this hybrid bilayer [52] that clearly distinguishes this system from QW-QW and graphene-graphene bilayers. In the former case, $\Lambda_{\mathbf{p p}^{\prime}} \rightarrow 1$, and the contact interactions drive Cooper pairing only in the s-wave channel. For the case of a graphene/graphene bilayer with a gapless spectrum, $\delta=0$, the factor must be modified as $\Lambda_{\mathbf{p p}^{\prime}} \rightarrow \Lambda_{\mathbf{p p}^{\prime}}^{2}$ [53]. As a result, there are three channels $l=0, \pm 1$, and the 
corresponding coupling constants are $\lambda_{0}=\nu_{0} V / 2$ and $\lambda_{ \pm 1}=\nu_{0} V / 4$. In a similar manner as for the QW-GaAs bilayer, the s-wave channel is the dominant Cooper pairing channel.

The origin of two competing channels can be tracked to the presence of the pseudospin degree of freedom for Dirac holes. In the sublattice basis, the order parameter $\hat{\Delta}_{\mathbf{p}}=\left\{\Delta_{\mathrm{p}}^{\mathrm{A}}, \Delta_{\mathrm{p}}^{\mathrm{B}}\right\}$ has two components: $\left(\Delta_{\mathrm{p}}^{\mathrm{A}}=\right.$ $-V\left\langle h_{\mathbf{p}}^{\mathrm{A} \dagger} e_{\mathbf{p}}\right\rangle$ and $\left.\Delta_{\mathbf{p}}^{\mathrm{B}}=-V\left\langle h_{\mathbf{p}}^{\mathrm{B} \dagger} e_{\mathbf{p}}\right\rangle\right)$. These components describe selective Cooper pairing with correlations only at one of two sublattices. These components are related to the order parameter $\Delta_{\mathrm{p}}^{\mathrm{v}}$ introduced above, as follows:

$$
\Delta_{\mathbf{p}}^{\mathrm{v}}=s_{\mathbf{p}} \Delta_{\mathbf{p}}^{\mathrm{A}} e^{-i \frac{\zeta \phi_{\mathbf{p}}}{2}}+c_{\mathbf{p}} \Delta_{\mathbf{p}}^{\mathrm{B}} e^{i \frac{\zeta \phi_{\mathbf{p}}}{2}}
$$

The order parameter in the sublattice picture has the only s-wave component and is therefore momentumindependent $\hat{\Delta}$. As a result, there is a one-to-one correspondence between channels in the band basis and components of the order parameter in the sublattice basis. The relation between them is valley-dependent and can be presented as

$$
\Delta_{-\zeta / 2}^{\mathrm{v}}=s_{\mathrm{F}} \Delta^{\mathrm{A}}, \quad \Delta_{\zeta / 2}^{\mathrm{v}}=c_{\mathrm{F}} \Delta^{\mathrm{B}}
$$

The basis choice is a matter of convenience, and in the remainder of the paper, we will deal only with the order parameter in the sublattice basis.

Thus far, we have considered only the instability toward Cooper pairing with zero momentum, $\mathbf{q}=0$. As we demonstrate below, the chiral nature of Dirac electrons results in intertwining between $\Delta^{\mathrm{A}}$ and $\Delta^{\mathrm{B}}$ at finite $\mathbf{q}$.

\section{GINZBURG-LANDAU HAMILTONIAN}

Above the transition temperature, the order parameter vanishes, but Gaussian fluctuations remain, which are usually interpreted as fluctuating CPs. These fluctuations are described by the dynamical bosonic field $\hat{\Delta}_{t \mathbf{r}}=\left\{\Delta_{t \mathbf{r}}^{\mathrm{A}}, \Delta_{t \mathbf{r}}^{\mathrm{B}}\right\}$, and their energetics are determined by the GL functional $F_{\mathrm{GL}}[\Delta]$, which is derived in Appendix A. After a proper rescaling [54], $F_{\mathrm{GL}}[\Delta]$ can be presented as

$$
F_{\mathrm{GL}}=\int \mathbf{d r} \Delta_{t \mathbf{r}}^{\dagger} \hat{H}_{\mathrm{GL}} \Delta_{t \mathbf{r}}
$$

The dimensionless Hermitian matrix $\hat{H}_{\mathrm{GL}}$ is the GL Hamiltonian. This matrix describes two bosonic modes $\left(\Delta_{t \mathbf{r}}^{\mathrm{A}}\right.$ and $\left.\Delta_{t \mathbf{r}}^{\mathrm{B}}\right)$ intertwined by effective pseudospin-orbit interactions as

$$
\hat{H}_{\mathrm{GL}}=\left(\begin{array}{cc}
\varepsilon_{\mathrm{A}}+\xi^{2} \mathbf{q}^{2} & -\xi_{\star}\left(\zeta q_{x}-i q_{y}\right) \\
-\xi_{\star}\left(\zeta q_{x}+i q_{y}\right) & \varepsilon_{\mathrm{B}}+\xi^{2} \mathbf{q}^{2}
\end{array}\right) .
$$

Each mode has a temperature-dependent energy gap $\varepsilon_{\mathrm{A}(\mathrm{B})}=\ln \left[T / T_{\mathrm{A}(\mathrm{B})}^{0}\right]$. The gap vanishes at the transition temperature $T_{\mathrm{A}(\mathrm{B})}^{0}$ given by Eq. (9) (combined with the channel labeling rule, Eq. (11)), which signals Cooper instability in the corresponding channel. The largest of them $T_{0}=\max \left[T_{\mathrm{A}}^{0}, T_{\mathrm{B}}^{0}\right]$ determines the critical temperature of electron-hole Cooper pairing. The lengths

$$
\xi=\sqrt{\frac{7 \zeta(3)}{2}} \frac{\hbar v_{\mathrm{e}} v_{\mathrm{h}}}{2 \pi\left(v_{\mathrm{e}}+v_{\mathrm{h}}\right) T}, \quad \xi_{\star}=\frac{\hbar v_{\mathrm{e}}}{4 \lambda\left(v_{\mathrm{e}}+v_{\mathrm{h}}\right) p_{\mathrm{F}}}
$$

scale the quadratic kinetic energy of the modes and their pseudospin-orbit interactions, respectively. Here, $\lambda=\max \left[\lambda_{\mathrm{A}}, \lambda_{\mathrm{B}}\right]$ is the coupling constant in the leading channel. The ratio of scales $\xi_{\star} / \xi \sim T_{0} / \lambda v_{\mathrm{h}} p_{\mathrm{F}}$ is small; thus, the contribution of the effective pseudospin-orbit interactions to the kinetic energy is small compared with the quadratic term.

The effective pseudospin-orbit interactions mix and intertwine components of the order parameter $\Delta_{\mathbf{q}}^{\mathrm{A}}$ and $\Delta_{\mathbf{q}}^{\mathrm{B}}$. The spectrum of the GL Hamiltonian $H_{\mathrm{GL}}$ has two eigenmodes $(\gamma= \pm 1)$ and is given by

$$
\varepsilon_{\gamma \mathbf{q}}=\varepsilon_{\mathrm{s}}+\xi^{2} \mathbf{q}^{2}+\gamma d_{\mathbf{q}}, \quad d_{\mathbf{q}}=\sqrt{\varepsilon_{\mathrm{z}}^{2}+\xi_{\star}^{2} \mathbf{q}^{2}} .
$$

Here, $\varepsilon_{\mathrm{S}}=\left(\varepsilon_{\mathrm{A}}+\varepsilon_{\mathrm{B}}\right) / 2$ is the average energy of the modes, and $\varepsilon_{\mathrm{z}}=\left(\varepsilon_{\mathrm{A}}-\varepsilon_{\mathrm{B}}\right) / 2$ can be interpreted as the effective Zeeman term in the GL Hamiltonian $H_{\mathrm{GL}}$. These terms are given by

$$
\varepsilon_{\mathrm{s}}=\ln \left(\frac{T}{\sqrt{T_{\mathrm{A}}^{0} T_{\mathrm{B}}^{0}}}\right), \quad \varepsilon_{\mathrm{z}}=\frac{1}{2} \ln \left(\frac{T_{\mathrm{B}}^{0}}{T_{\mathrm{A}}^{0}}\right) .
$$

The average energy $\varepsilon_{\mathrm{s}}$ smoothly decreases with temperature, but the effective Zeeman term $\varepsilon_{z}$ is temperatureindependent. Its sign and magnitude

$$
\varepsilon_{z}=\frac{\lambda_{\mathrm{B}}-\lambda_{\mathrm{A}}}{2 \lambda_{\mathrm{A}} \lambda_{\mathrm{B}}} \approx \frac{1}{\sqrt{\lambda_{\mathrm{A}} \lambda_{\mathrm{B}}}} \frac{\delta}{\epsilon_{\mathrm{F}}^{\mathrm{h}}}
$$

are given by the sublattice asymmetry $\delta$ and vanish if $\delta=0$.

If the effective Zeeman term is smaller $\epsilon_{\mathrm{z}}<\epsilon_{\mathrm{M}}$ than $\epsilon_{\mathrm{M}}=\xi_{\star}^{2} / 2 \xi^{2}$, the effective spin-orbit interactions reshape the parabolic lower eigenmode $\varepsilon_{-, \mathbf{q}}$ into a Mexican-hat curve, as presented in Fig. 2-a. A minimum occurs at finite momentum $q_{\mathrm{M}}$ with an energy gain of $\Delta \varepsilon_{\mathrm{M}}$ with respect to $\varepsilon_{-, \mathbf{q}}$ at $\mathbf{q}=0$, as follows:

$$
\xi q_{\mathrm{M}}=\frac{\xi_{\star}}{\xi} \frac{\sqrt{\varepsilon_{\mathrm{M}}^{2}-\varepsilon_{z}^{2}}}{2 \varepsilon_{\mathrm{M}}}, \quad \Delta \varepsilon_{\mathrm{M}}=-\frac{\left(\varepsilon_{\mathrm{M}}-\left|\varepsilon_{\mathrm{z}}\right|\right)^{2}}{2 \varepsilon_{\mathrm{M}}} .
$$

As a result, the electron-hole bilayer is unstable toward Cooper pairing with finite momentum (Fulde-Ferrell state [55]) or a non-uniform state (Larkin-Ovchinnikov state [56]). The nature of the condensed state can be addressed within the GL theory (with quartic terms in the free energy functional $F_{\mathrm{GL}}$ ), but such an analysis is beyond the scope of the present paper, which focuses on fluctuating CPs. It should be noted that the 


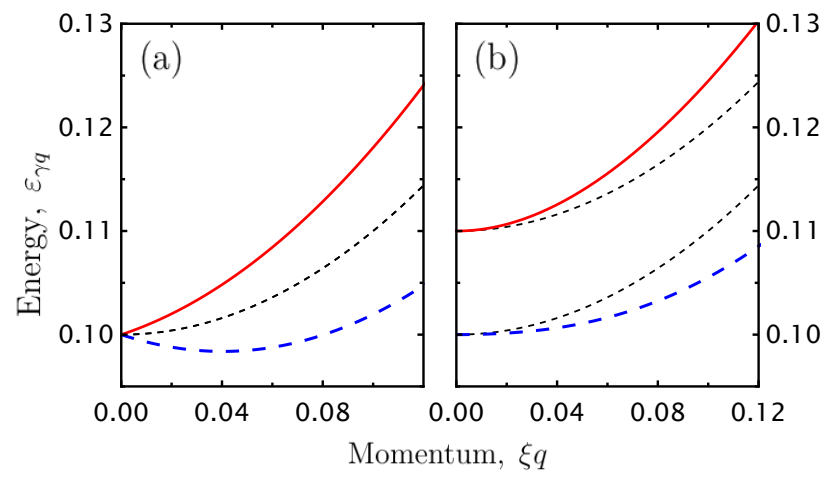

FIG. 2. Dispersion relation for fluctuating CPs at $\epsilon_{\mathrm{z}}=0$ (a) and $\epsilon_{\mathrm{z}}=0.1$ (b) for $\xi_{\star} / \xi=0.08$. The dashed black lines correspond to the spectrum without pseudospin-orbit interactions, $\xi_{\star} / \xi=0$. For a small asymmetry $\epsilon_{\mathrm{z}}<\varepsilon_{\mathrm{M}}$ between sublattices, the effective pseudospin-orbit interactions reshape the lower parabolic eigenmode into a Mexican-hatshaped curve. As a result, the system is unstable toward the Cooper pairing state with finite momentum (Fulde-FerrellLarkin-Ovchinnikov state).

FFLO phases are stabilized solely by the pseudospinorbit interactions and do not require the electron-hole density imbalance $[57,58]$. Since contribution of effective pseudospin-orbit interactions to the kinetic energy is small compared with the usual quadratic term, this regime $\epsilon_{\mathrm{z}}<\epsilon_{\mathrm{M}}$ is realized only at vanishingly small energy asymmetry $\delta$ between graphene sublattices.

If the effective Zeeman term is sufficiently large $\left(\epsilon_{\mathrm{z}}>\right.$ $\left.\epsilon_{\mathrm{M}}\right)$ the effective pseudospin-orbit interactions impact the slope of the dispersion curves $\varepsilon_{\gamma \mathbf{q}}$ presented in Fig. 2-b, but do not reshape them.

The intertwining between $\Delta_{t \mathbf{r}}^{\mathrm{A}}$ and $\Delta_{t \mathbf{r}}^{\mathrm{B}}$ is manifested in the nontrivial geometry and topology of the $H_{\mathrm{GL}}$ spectrum. The nontrivial geometry is intricately related to the momentum space texture for the unit vector $\mathbf{n}_{\mathbf{q}}=\mathbf{h}_{\mathbf{q}} /\left|\mathbf{h}_{\mathbf{q}}\right|$ defined within the parametrization of the GL Hamiltonian $H_{\mathrm{GL}}(\mathbf{q})=\hat{1} \cdot h_{\mathbf{q}}^{0}+\hat{\boldsymbol{\sigma}}_{\mathrm{P}} \cdot \mathbf{h}_{\mathbf{q}}$ in terms of Pauli matrices $\hat{\boldsymbol{\sigma}}_{\mathrm{P}}$. The vector $\mathbf{n}_{\mathbf{q}}$ follows the topological momentum space meron texture. Depending on the sign of $\epsilon_{\mathrm{z}}$, the vector points up or down at the momentum ori$\operatorname{gin} \mathbf{q}=0$. For a large momentum $\mathbf{q}$, the vector $\mathbf{n}_{\mathbf{q}}$ lies in the horizontal plane and follows the vortex-like texture. Being projected to the Riemann sphere, the vector field spans the half that dictates the topological Chern number to be a half-integer $\left|\mathcal{C}_{\gamma}^{\mathrm{C}}\right|=1 / 2$.

In formal mathematics, the nontrivial geometry of the $H_{\mathrm{GL}}$ spectrum can be characterized by the generalized Berry connection $\hat{\mathcal{A}}_{\mathbf{q}}$, which also includes the off-diagonal matrix elements, as follows:

$$
\mathcal{A}_{\mathbf{q}}^{\gamma \gamma^{\prime}}={ }_{\mathrm{C}}\left\langle\gamma \mathbf{q}\left|i \nabla_{\mathbf{q}}\right| \gamma^{\prime} \mathbf{q}\right\rangle_{\mathrm{C}}, \quad T_{\gamma \mathbf{q}}^{\alpha \beta}=\mathcal{A}_{\mathbf{q} \alpha}^{\gamma \bar{\gamma}} \mathcal{A}_{\mathbf{q} \beta}^{\bar{\gamma} \gamma}
$$

Here, we have also introduced the quantum geometric tensor $\hat{T}_{\gamma \mathbf{q}}$ for fluctuating CPs with $\alpha$ and $\beta$ as Cartesian indices $(x$ and $y)$ [59-61]. The real part of the tensor defines the quantum metric $\hat{G}_{\gamma \mathbf{q}}=\operatorname{Re}\left[\hat{T}_{\gamma \mathbf{q}}\right]$, which allows one to measure distances between eigenstates $|\gamma \mathbf{q}\rangle_{\mathrm{C}}$ in momentum space. The quantum metric for fluctuating CPs is given by

$$
\begin{array}{r}
\hat{G}_{\gamma \mathbf{q}}=\frac{1}{8 d_{\mathbf{q}}^{4}}\left[\xi _ { \star } ^ { 4 } \mathbf { q } ^ { 2 } \left[\sigma_{\mathrm{P}}^{\mathrm{z}} \cos \left(2 \phi_{\mathbf{q}}\right)\right.\right. \\
\left.+\sigma_{\mathrm{P}}^{\mathrm{x}} \sin \left(2 \phi_{\mathbf{q}}\right)\right] \\
\left.-\xi_{\star}^{2}\left(d_{\mathbf{q}}^{2}+\varepsilon_{\mathrm{z}}^{2}\right)\right] .
\end{array}
$$

The second term is independent of the direction of momentum q, and the first term has quadrupole symmetry. The imaginary part of the quantum geometric tensor for fluctuating CPs defines their nontrivial Berry curvature $\Omega_{\gamma \mathbf{q}}^{\mathrm{C}}=-2 \epsilon_{\alpha \beta z} \operatorname{Im}\left[\hat{T}_{\gamma \mathbf{q}}^{\alpha \beta}\right]$, which matches with the another definition $\Omega_{\gamma \mathbf{q}}^{\mathrm{C}}=\left[\boldsymbol{\nabla}_{\mathbf{q}} \times \mathcal{A}_{\gamma \mathbf{q}}^{\gamma \gamma}\right]_{\mathrm{z}}$. The Berry curvature has the opposite sign for two eigenmodes, is valleydependent, and is given by

$$
\Omega_{\gamma \mathbf{q}}^{\mathrm{C}}=\frac{\zeta \gamma \epsilon_{\mathrm{z}}}{d_{\mathbf{q}}^{\frac{3}{2}}}, \quad \mathcal{C}_{\gamma}^{\mathrm{C}}=\int \frac{d \mathbf{q}}{2 \pi} \Omega_{\gamma \mathbf{q}}^{\mathrm{C}}=\frac{\zeta \gamma \varepsilon_{z}}{2\left|\varepsilon_{z}\right|} .
$$

Being inherited from the Dirac holes, the band geometry is also characterized by the half-integer Chern number $\mathcal{C}_{\gamma}^{\mathrm{C}}$. Therefore, we interpret the hybrid electron-hole fluctuating CPs to be topologically nontrivial.

The nontrivial geometries of fluctuating CPs closely follow the geometry for Dirac holes in graphene. However, there are two major differences between Dirac holes and fluctuating CPs. First, the pair of massive Dirac bands is separated by the global gap (i.e., present for all momenta), which is not the case for fluctuating $\mathrm{CP}$ eigenmodes. Besides the existence of bands with nontrivial Chern numbers, the stability of the edge modes at boundaries and domain walls also requires a global energy gap (in the Discussions section, we discuss approaches to overcome this obstacle). However, the absence of this gap is not an obstacle for anomalous transport phenomena, which rely on the nonzero Berry curvature of eigenmodes and will be considered in the following sections. Second, fluctuating CPs are not bosonic quasiparticles (with Hermitian Hamiltonian dynamics) but overdamped bosonic modes. For this reason, the corresponding anomalous transport phenomena are outside the range of present theories [62-64].

\section{KINETIC EQUATION}

The dynamics of fluctuating CPs are governed by the time-dependent GL (TDGL) equation (see Ref. [38] and Appendix C). Components of fluctuating CPs have opposite charges and are spatially separated. For these reasons, coupling with electric potentials in both layers $\left(\phi_{t \mathbf{r}}^{\mathrm{e}}\right.$ 
and $\left.\phi_{t \mathbf{r}}^{\mathrm{h}}\right)$ can be introduced via the Peierls substitution $\partial_{t} \rightarrow \partial_{t}+i e \phi_{t \mathbf{r}}^{\mathrm{eh}}$ with $\phi_{t \mathbf{r}}^{\mathrm{eh}}=\phi_{t \mathbf{r}}^{\mathrm{e}}-\phi_{t \mathbf{r}}^{\mathrm{h}}$. As a result, the TDGL equation can be presented as follows:

$$
\begin{aligned}
\tau^{*}\left(\partial_{t}+i e \phi_{t \mathbf{r}}^{\mathrm{eh}}\right) \Delta & =-\hat{H}_{\mathrm{GL}} \Delta+\eta_{t \mathbf{r}}, \\
\left\langle\eta_{t \mathbf{r}} \eta_{t^{\prime} \mathbf{r}^{\prime}}^{\dagger}\right\rangle & =2 T \tau^{\prime} \hat{1} \delta_{t t^{\prime}} \delta_{r \mathbf{r}^{\prime}} .
\end{aligned}
$$

Here, $\tau=\tau^{\prime}+i \tau^{\prime \prime}$, where $\tau^{\prime}=\pi \hbar / 8 T$ gives the dissipation rate of fluctuating CPs and $\tau^{\prime \prime}=\hbar / \lambda\left(v_{\mathrm{e}}+v_{\mathrm{h}}\right) p_{\mathrm{F}}$ weights the Hermitian part of their dynamics. The external complex field $\eta_{t \mathbf{r}}=\left\{\eta_{t \mathbf{r}}^{\mathrm{A}}, \eta_{t \mathbf{r}}^{\mathrm{B}}\right\}$ is the Langevin noise. Its presence is dictated by the fluctuation-dissipation theorem, and the correlation function $\left\langle\eta_{t \mathbf{r}} \eta_{t^{\prime} \mathbf{r}^{\prime}}^{\dagger}\right\rangle$, which is free of temporal and spatial correlations (white noise), is proportional to the relaxation rate $\tau^{\prime}$ but does not depend on $\tau^{\prime \prime}$. In the considered weak-to-moderate Cooper pairing regime, their ratio is small $\tau^{\prime \prime} / \tau^{\prime} \sim T / \lambda\left(v_{\mathrm{e}}+v_{\mathrm{h}}\right) p_{\mathrm{F}}$; hence, the dissipation of fluctuating CPs is an essential component in their dynamics. These CPs cannot be interpreted as bosonic quasiparticles, but are instead overdamped bosonic modes.

The contribution of fluctuating CPs in transport phenomena can be addressed by applying the linear response approach to the TDGL equation. These calculations are presented in Appendix B. In the main part of this paper, we follow another route. As it is presented in Appendix C, the TDGL equation, Eq. (21), can be transformed into a kinetic equation for the distribution function of fluctuating $\operatorname{CPs} n_{\gamma}(\mathbf{R}, \mathbf{q})$, which is given by:

$$
\begin{array}{r}
\partial_{t} n_{\gamma}+e \mathbf{E}^{\mathrm{eh}} \partial_{\mathbf{q}} n_{\gamma}+\frac{\tau^{\prime \prime}}{|\tau|^{2}}\left(\partial_{\mathbf{q}} \varepsilon_{\gamma \mathbf{q}}+\mathbf{u}_{\gamma \mathbf{q}}^{\Omega}+\right. \\
\left.\mathbf{u}_{\gamma \mathbf{q}}^{\mathrm{G}}\right) \partial_{\mathbf{R}} n_{\gamma}=-\frac{2 \varepsilon_{\gamma \mathbf{q}} \tau^{\prime}}{|\tau|^{2}}\left(n_{\gamma}-n_{\gamma}^{0}\right) .
\end{array}
$$

Here $n_{\gamma_{\mathbf{q}}}^{0}=T / \varepsilon_{\gamma_{\mathbf{q}}}$ is the equilibrium classical distribution function for fluctuating CPs. We have introduced two distinct anomalous velocities $\mathbf{u}_{\gamma \mathbf{q}}^{\Omega}$ and $\mathbf{u}_{\gamma \mathbf{q}}^{\mathrm{G}}$, which are generated by the nonzero Berry curvature $\Omega_{\gamma \mathbf{q}}$ and quantum metric $\hat{G}_{\gamma \mathbf{q}}$, respectively. Their explicit expressions are given by

$$
\begin{array}{r}
\mathbf{u}_{\gamma_{\mathbf{q}}}^{\Omega}=\Omega_{\gamma \mathbf{q}}\left[\mathbf{e}_{\mathrm{z}} \times e \mathbf{E}^{\mathrm{eh}}\right] \frac{\tau^{\prime \prime}\left(\varepsilon_{\gamma_{\mathbf{q}}}-\varepsilon_{\bar{\gamma} \mathbf{q}}\right)^{2}|\tau|^{2}}{\left|\varepsilon_{\gamma_{\mathbf{q}}} \tau^{*}+\varepsilon_{\bar{\gamma} \mathbf{q}} \tau\right|^{2}}, \\
\mathbf{u}_{\gamma \mathbf{q}}^{\mathrm{G}}=\hat{G}_{\gamma \mathbf{q}} \cdot e \mathbf{E}^{\mathrm{eh}} \frac{\tau^{\prime}\left(\varepsilon_{\gamma \mathbf{q}}^{2}-\varepsilon_{\bar{\gamma} \mathbf{q}}^{2}\right)|\tau|^{2}}{\left|\varepsilon_{\gamma \mathbf{q}} \tau^{*}+\varepsilon_{\bar{\gamma} \mathbf{q}} \tau\right|^{2}} .
\end{array}
$$

The nontrivial Berry curvature is known to generate an anomalous velocity in fermionic and bosonic systems [1]. The presence of an additional anomalous velocity $\mathbf{u}_{\gamma \mathbf{q}}^{\mathrm{G}}$ is a unique feature of fluctuating $\mathrm{CP}$ dynamics and is intricately related to their dissipative nature. If we omit the dissipation and rescale the time $\left(\tau^{\prime}=0\right.$ and $\left.t \rightarrow \tau^{\prime \prime} t\right)$, the TDGL equation reduces to a Schrodingerlike equation $i \partial_{t} \Delta=\hat{H}_{\mathrm{GL}} \Delta$. At this limit, the additional term $\mathbf{u}_{\gamma \mathbf{q}}^{\mathrm{G}}=0$ vanishes, and the conventional expression $\mathbf{u}_{\gamma \mathbf{q}}^{\Omega}=\Omega_{\gamma \mathbf{q}}\left[\mathbf{e}_{\mathrm{z}} \times e \mathbf{E}^{\mathrm{eh}}\right]$ for the anomalous velocity is recovered.

The derived kinetic equation for fluctuating CPs, Eq. (22), is the main result of this section and is a key to the anomalous transport phenomena mediated by fluctuating CPs. In Appendix C, we demonstrate that resulting predictions agree with ones derived by applying the linear response approach to the TDGL equation.

\section{PARACONDUCTIVITY}

Due to the spatial separation of electrons and holes forming fluctuating CPs, their motion induces electric currents in both layers. These currents have opposite directions but the same magnitude (as well as the same conductivity and transconductivity, which is defined as the response of an electric current in one layer to an electric field in another layer). The electric field difference $\mathbf{E}^{\mathrm{eh}}$ shifts the distribution function $n_{\gamma \mathbf{q}}^{0} \rightarrow n_{\gamma_{\mathbf{q}}}^{0}+n_{\gamma_{\mathbf{q}}}^{1}$ and induces electric currents in both layers as

$$
\mathbf{J}^{\mathrm{e}(\mathrm{h})}= \pm \sum_{\mathbf{p} \gamma} \partial_{\mathbf{q}} \varepsilon_{\gamma \mathbf{q}} n_{\gamma \mathbf{q}}^{1}, \quad n_{\gamma \mathbf{q}}^{1}=-\frac{|\tau|^{2} \mathbf{E}^{\mathrm{eh}} \partial_{\mathbf{q}} n_{\gamma \mathbf{q}}^{0}}{2 \tau^{\prime} \varepsilon_{\gamma \mathbf{q}}} .
$$

The electric currents are parallel to $\mathbf{E}^{\mathrm{eh}}$, and the corresponding contribution to the longitudinal conductivity represents the sum of conventional Aslamazov-Larkin paraconductivities for two pairing channels. Besides, the electric field difference $\mathbf{E}^{\mathrm{eh}}$ induces an additional contribution via the anomalous velocities as

$$
\mathbf{J}^{\mathrm{e}(\mathrm{h})}= \pm \sum_{\gamma \mathbf{q}}\left(u_{\gamma \mathbf{q}}^{\Omega}+u_{\gamma \mathbf{q}}^{\mathrm{G}}\right) n_{\gamma \mathbf{q}}^{0}
$$

These two terms can be interpreted as anomalous Aslamazov-Larkin paraconductivities. The velocity $u_{\gamma \mathbf{q}}^{\Omega}$ is perpendicular to the electric field difference $\mathbf{E}^{\mathrm{eh}}$ and is responsible for the anomalous contribution to the Hall conductivity. The velocity $u_{\gamma_{\mathbf{q}}}^{\mathrm{G}}$ has both components, which are perpendicular/parallel to the electric field difference $\mathbf{E}^{\mathrm{eh}}$. However, the off-diagonal elements of the quantum metric $\hat{G}_{\gamma \mathbf{q}}$ given by Eq. (19) have quadrupole symmetry and vanish over angle integration. As a result, only the second term in Eq. (19) survives, and the quantum-metric-induced anomalous velocity $u_{\gamma_{\mathbf{q}}}^{\mathrm{G}}$ provides an additional contribution only to the longitudinal conductivity. An explicit evaluation of the different contributions to the conductivity is presented in Appendix B. To the leading order in $\xi_{\star} / \xi$ and $\tau^{\prime \prime} / \tau^{\prime}$ (it should be noted that within this approximation the effect of the effective pseudospin-orbit interactions at the dispersion of fluctuating CPs is neglected), the expressions $\left(\sigma_{\mathrm{L}} \equiv \sigma_{x x}\right.$ 
and $\left.\sigma_{\mathrm{H}} \equiv \sigma_{x y}\right)$ are given by

$$
\begin{array}{r}
\sigma_{\mathrm{L}}^{\mathrm{AL}}=\frac{e^{2}}{2 \pi \hbar} F_{\mathrm{AL}}, \quad \sigma_{\mathrm{L}}^{\mathrm{G}}=\frac{e^{2}}{2 \pi \hbar}\left(\frac{\xi_{\star}}{\xi}\right)^{2} F_{\mathrm{G}}, \\
\sigma_{\mathrm{H}}^{\Omega}=\zeta \frac{e^{2}}{2 \pi \hbar}\left(\frac{\xi_{\star}}{\xi}\right)^{2} \frac{\tau^{\prime \prime}}{\tau^{\prime}} F_{\Omega} .
\end{array}
$$

The temperature behaviors of these terms are governed by dimensionless functions $F \equiv F\left(\varepsilon_{\mathrm{A}}, \varepsilon_{\mathrm{B}}\right)$. The latter terms depend only on $\varepsilon_{\mathrm{A}}=\ln \left[T / T_{\mathrm{A}}^{0}\right]$ and $\varepsilon_{\mathrm{B}}=\ln \left[T / T_{\mathrm{B}}^{0}\right]$ and are given by

$$
\begin{array}{r}
F_{\mathrm{AL}}=\frac{\pi}{32}\left(\frac{1}{\varepsilon_{\mathrm{A}}}+\frac{1}{\varepsilon_{\mathrm{B}}}\right), \\
F_{\mathrm{G}}=\frac{\pi}{8\left(\varepsilon_{\mathrm{A}}-\varepsilon_{\mathrm{B}}\right)^{2}} \ln \left[\frac{\left(\varepsilon_{\mathrm{A}}+\varepsilon_{\mathrm{B}}\right)^{2}}{4 \varepsilon_{\mathrm{A}} \varepsilon_{\mathrm{B}}}\right], \\
F_{\Omega}=\frac{\pi}{8\left(\varepsilon_{\mathrm{A}}-\varepsilon_{\mathrm{B}}\right)}\left\{\frac{\ln \left[\varepsilon_{\mathrm{A}} / \varepsilon_{\mathrm{B}}\right]}{\varepsilon_{\mathrm{A}}-\varepsilon_{\mathrm{B}}}-\frac{2}{\varepsilon_{\mathrm{A}}+\varepsilon_{\mathrm{B}}}\right\} .
\end{array}
$$

Without loss of generality, we can assume that $T_{\mathrm{A}}^{0}>T_{\mathrm{B}}^{0}$. It is instructive to introduce the dimensionless temperature $T / T_{\mathrm{A}}^{0}$ and the relation of transition temperatures for two channels $T_{\mathrm{B}}^{0} / T_{\mathrm{A}}^{0}$. The corresponding dependence for the $F$-functions is presented in Fig. 3.

The factor $F_{\mathrm{AL}}$ represents a sum of two independent terms describing the conventional Aslamazov-Larkin effect for each of two competing channels. This factor reaches a maximum when two channels are degenerate $\left(T_{\mathrm{A}}^{0}=T_{\mathrm{B}}^{0}\right)$, and the corresponding result is two times larger than that for the case in which only one channel is present (e.g., $T_{\mathrm{B}}^{0} / T_{\mathrm{A}}^{0} \rightarrow 0$ ). In the vicinity of the transition temperature $T_{0}$, the factor $F_{\mathrm{AL}} \sim\left(T-T_{0}\right)^{-1}$ exhibits critical behavior, which, for the case of degeneracy, can be presented as

$$
F_{\mathrm{AL}}=\frac{\pi}{16} \frac{1}{\ln \left[T / T_{0}\right]} \rightarrow \frac{\pi}{16} \frac{T_{0}}{T-T_{0}},
$$

and diverges at the transition temperature.

The term $F_{\mathrm{G}}$ relies on the channel intertwining and also reaches a maximum when the two channels are degenerate. In this case, the expression for $F_{\mathrm{G}}$ has a stronger divergence

$$
F_{\mathrm{G}}=\frac{\pi}{32} \frac{1}{\ln ^{2}\left[T / T_{0}\right]} \rightarrow \frac{\pi}{32}\left(\frac{T_{0}}{T-T_{0}}\right)^{2}
$$

than the value $F_{\mathrm{AL}}$ given by Eq. (28). As a result, $F_{\mathrm{G}}$ exceeds $F_{\mathrm{AL}}$ in the broad parameter range around the degeneracy point. However, the corresponding term $\sigma_{\mathrm{L}}^{\mathrm{G}}$ in Eq. (26) has a small prefactor $\left(\xi_{\star} / \xi\right)^{2}$, which causes the anomalous contribution $\sigma_{L}^{\mathrm{G}}$ to be small compared with the conventional contribution $\sigma_{L}^{\mathrm{AL}}$.

The factor $F_{\Omega}$ also relies on channel intertwining, but vanishes when the two channels are degenerate, $F_{\Omega}=0$. The Berry curvatures for fluctuating CPs have opposite

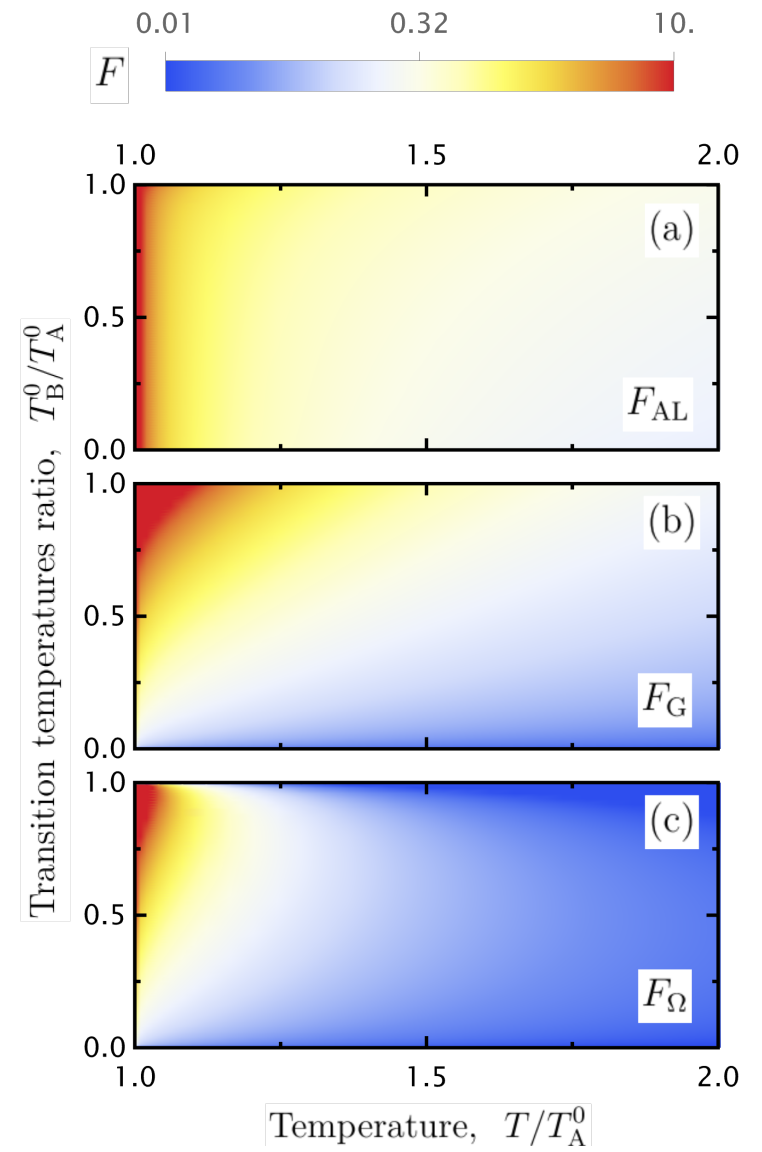

FIG. 3. Dependence of the $F$-functions introduced in Eq. (27) on temperature $T / T_{\mathrm{A}}^{0}$ and the ratio between transition temperatures $T_{\mathrm{B}}^{0} / T_{\mathrm{A}}^{0}$ for two competing pairing channels. Both conventional and anomalous contributions to the conductivity tensor exhibit critical behavior and diverge at the transition temperature.

signs for the two modes, and their contributions vanish when the two modes have the same thermal population. As a result, the factor $F_{\Omega}$ reaches a maximum for finite but small splitting between transition temperatures for two channels.

\section{TRANSPORT PHENOMENA}

The interplay of the conventional Aslamazov-Larkin effect and the effect of Coulomb drag due to momentum transfer between layers has already been discussed in detail. Here, we discuss possible experimental setups that can be used to observe the anomalous Aslamazov-Larkin contributions to the conductivity (and transconductivity) tensor mediated by fluctuating $\mathrm{CPs}$ with nontrivial geometries.

First, we recall that the anomalous contribution $\sigma_{\mathrm{L}}^{\mathrm{G}}$, which originates from the quantum geometric tensor for 
fluctuating CPs, is small compared with $\sigma_{\mathrm{L}}^{\mathrm{AL}}$; thus, this contribution is difficult to observe unless $\xi_{\star}$ is comparable to $\xi$. For this reason, we focus on the transverse anomalous contribution $\sigma_{\mathrm{H}}^{\Omega}$ that arises from the nonzero Berry curvature for fluctuating CPs. The sign of the contribution is valley-dependent, and no current appears [65] until the symmetry between valleys is broken. In this section, we consider a regime of ultimate asymmetry, in which holes from only one of two valleys participate in fluctuating CPs. We discuss possible approaches to achieve this regime in the Discussions section.

Second, we note the presence of single-particle contributions to the Hall conductivity of graphene $\left(\sigma_{\mathrm{xy}}^{\mathrm{h}}=\right.$ $\zeta e^{2} \delta / 2 \pi \hbar\left|\epsilon_{\mathrm{F}}^{\mathrm{h}}\right|$ per valley in the absence of valley asymmetry). This contribution can easily exceed the small contribution mediated by fluctuating CPs. For this reason, we consider only experimental setups with a voltage probe in a semiconductor QW layer, where electrons are free of nontrivial geometries. The three possible setups are sketched in Fig. 4.

The transport phenomena in an electron-hole bilayer can be described by utilizing a generalized conductivity matrix $\hat{\sigma}$, which connects currents $\hat{J}=\hat{\sigma} \hat{E}$ in two layers $J=\left\{J_{x}^{\mathrm{e}}, J_{y}^{\mathrm{e}}, J_{y}^{\mathrm{h}}, J_{y}^{\mathrm{h}}\right\}$ with the corresponding electric fields $E=\left\{E_{x}^{\mathrm{e}}, E_{y}^{\mathrm{e}}, E_{y}^{\mathrm{h}}, E_{y}^{\mathrm{h}}\right\}$. If we recall that fluctuating CPs are excited by an electric field difference $\mathbf{E}^{\mathrm{eh}}$ and their motion generates opposite currents in two layers, the conductivity matrix can be presented as

$$
\hat{\sigma}=\left(\begin{array}{cccc}
\sigma_{\mathrm{L}}^{\mathrm{e}}+\sigma_{\mathrm{L}}^{\mathrm{C}} & -\sigma_{\mathrm{H}}^{\mathrm{C}} & -\sigma_{\mathrm{D}}-\sigma_{\mathrm{L}}^{\mathrm{C}} & \sigma_{\mathrm{H}}^{\mathrm{C}} \\
\sigma_{\mathrm{H}}^{\mathrm{C}} & \sigma_{\mathrm{L}}^{\mathrm{e}}+\sigma_{\mathrm{L}}^{\mathrm{C}} & -\sigma_{\mathrm{H}}^{\mathrm{C}} & -\sigma_{\mathrm{D}}-\sigma_{\mathrm{L}}^{\mathrm{C}} \\
-\sigma_{\mathrm{D}}-\sigma_{\mathrm{L}}^{\mathrm{C}} & \sigma_{\mathrm{H}}^{\mathrm{C}} & \sigma_{\mathrm{L}}^{\mathrm{h}}+\sigma_{\mathrm{L}}^{\mathrm{C}} & -\sigma_{\mathrm{H}}^{\mathrm{h}}-\sigma_{\mathrm{H}}^{\mathrm{C}} \\
-\sigma_{\mathrm{H}}^{\mathrm{C}} & -\sigma_{\mathrm{D}}-\sigma_{\mathrm{L}}^{\mathrm{C}} & \sigma_{\mathrm{H}}^{\mathrm{h}}+\sigma_{\mathrm{H}}^{\mathrm{C}} & \sigma_{\mathrm{L}}^{\mathrm{h}}+\sigma_{\mathrm{L}}^{\mathrm{C}}
\end{array}\right) .
$$

Here, $\sigma_{\mathrm{L}}^{\mathrm{e}(\mathrm{h})}$ is the conductivity of the electrons (holes), and $\sigma_{\mathrm{D}}$ is the contribution to the transconductivity due to momentum transfer between layers. $\sigma_{\mathrm{H}}^{\mathrm{h}}$ is the anomalous conductivity of Dirac holes, which is nonzero if the symmetry between valleys is broken. $\sigma_{\mathrm{L}}^{\mathrm{C}}=\sigma_{\mathrm{L}}^{\mathrm{AH}}+\sigma_{\mathrm{L}}^{\mathrm{G}}$ and $\sigma_{\mathrm{H}}^{\mathrm{C}}=\sigma_{\mathrm{H}}^{\Omega}$ are contributions of fluctuating CPs in the conductivity tensor. It is instructive to discuss the setups sketched in Fig. 4 separately.

For the setup shown in Fig. 5-a, an electric current is induced in the semiconductor QW. The corresponding Hall resistance of the QW is given by

$$
\rho_{\mathrm{H}}^{\mathrm{ee}}=\frac{E_{\mathrm{y}}^{\mathrm{e}}}{I_{\mathrm{x}}^{\mathrm{e}}}=-\frac{\sigma_{\mathrm{H}}^{\mathrm{C}}}{\left(\sigma_{\mathrm{L}}^{\mathrm{e}}\right)^{2}},
$$

which is proportional to the anomalous AslamazovLarkin paraconductivity mediated by fluctuating CPs. Importantly, this scheme represents a smoking gun experiment, but does not require independent electrical contacts for the two layers (which are essential for Coulomb drag measurements). Apparently, the anomalous Hall effect in a semiconductor QW is induced by

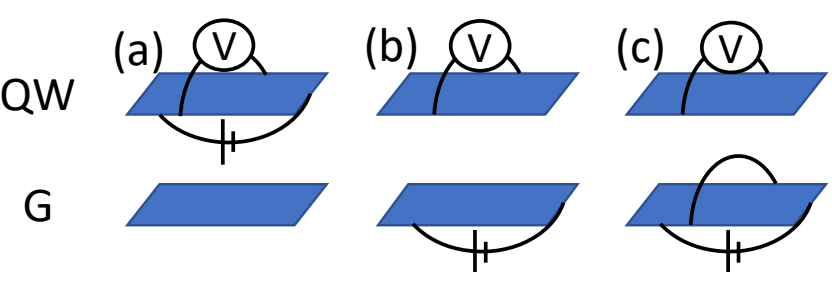

FIG. 4. Possible experimental setups with different connections to an external circuit. As discussed in the main text, setups (a) and (c) directly probe the transverse anomalous Aslamazov-Larkin paraconductivity mediated by fluctuating CPs, but this is not the case for setup (b).

non-perturbative interlayer correlations with chiral Dirac electrons in graphene.

For the setup shown in Fig. 4-b, the electric current is induced in graphene. At first glance, this setup appears to be convenient for probing transverse anomalous paraconductivity, but this is actually not the case. The corresponding Hall drag resistance is given by

$$
\bar{\rho}_{\mathrm{H}}^{\mathrm{he}}=\frac{E_{\mathrm{y}}^{\mathrm{e}}}{I_{\mathrm{x}}^{\mathrm{h}}}=\frac{\sigma_{\mathrm{H}}^{\mathrm{C}}}{\sigma_{\mathrm{L}}^{\mathrm{e}} \sigma_{\mathrm{L}}^{\mathrm{h}}}-\frac{\sigma_{\mathrm{D}}}{\sigma_{\mathrm{L}}^{\mathrm{e}} \sigma_{\mathrm{L}}^{\mathrm{h}}} \frac{\sigma_{\mathrm{H}}^{\mathrm{h}}}{\sigma_{\mathrm{L}}^{\mathrm{h}}},
$$

which has an additional term with the opposite sign. This term is nonzero even in the absence of fluctuating CPs. This resistance originates from the interplay between the anomalous Hall effect in graphene and the conventional longitudinal Coulomb effect due to momentum transfer between layers. The former results in transverse charge accumulation and momentum flow in graphene, while the latter induces a current in the semiconductor QW.

The transverse accumulation of charge carriers at graphene sides can be avoided by applying a shortcut, as shown for the setup in Fig. 4-c. As a result, the Hall drag resistance

$$
\rho_{\mathrm{H}}^{\mathrm{he}}=\frac{E_{\mathrm{y}}^{\mathrm{e}}}{I_{\mathrm{x}}^{\mathrm{h}}}=\frac{\sigma_{\mathrm{H}}^{\mathrm{C}}}{\sigma_{\mathrm{L}}^{\mathrm{e}} \sigma_{\mathrm{L}}^{\mathrm{h}}}
$$

has a single term, which is proportional to the anomalous Hall paraconductivity mediated by fluctuating CPs. It should be noted that this term has the same temperature dependence (up to a prefactor) as the Hall resistivity of QWs, given by Eq. (30). Observations of consistent temperature behavior would be strong evidence of an anomalous Hall response mediated by fluctuating CPs with nontrivial geometries.

\section{DISCUSSIONS}

For estimations, we use a set of parameters that are relevant for the hybrid graphene-GaAs bilayer reported 


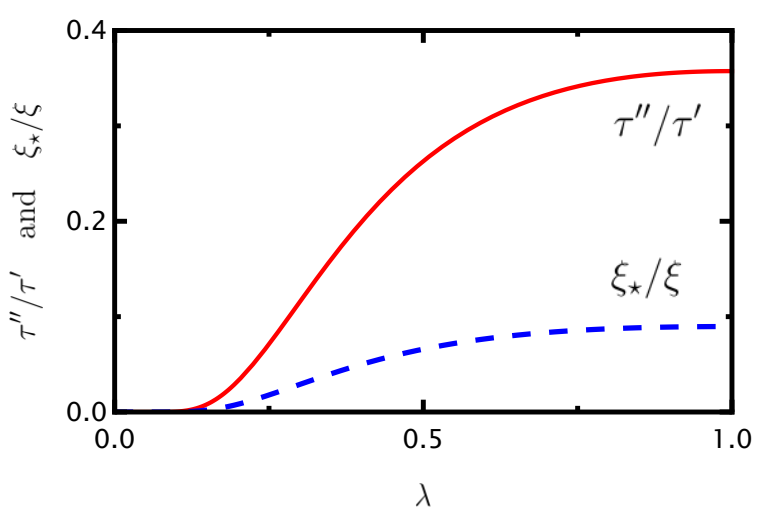

FIG. 5. Dependence of length $\xi_{\star} / \xi$ and time $\tau^{\prime \prime} / \tau^{\prime}$ scale ratios on the dimensionless coupling constant $\lambda$. Being small at $\lambda \ll 1$, the ratios grow drastically with coupling strength. However, being derived within the weak coupling theory, the values for the scale ratios at $\lambda \sim 1$ can only be used for guidance.

in Ref. [46]. We chose different densities for electrons $n_{\mathrm{e}} \approx 1.210^{11} \mathrm{~cm}^{-2}$ and holes $n_{\mathrm{h}} \approx 2.410^{11} \mathrm{~cm}^{-2}$ because holes have an additional valley degree of freedom. As a result, the Fermi momenta $p_{\mathrm{F}}$ for charge carriers in both layers are the same, but the Fermi energies $\left(\epsilon_{\mathrm{F}}^{\mathrm{h}} \approx 57 \mathrm{meV}\right.$ and $\left.\epsilon_{\mathrm{F}}^{\mathrm{e}} \approx 3.8 \mathrm{meV}\right)$ and Fermi velocities $\left(v_{\mathrm{e}} \approx 1.510^{7} \mathrm{~cm} / \mathrm{s}\right.$ and $\left.v_{\mathrm{h}} \approx 10^{8} \mathrm{~cm} / \mathrm{s}\right)$ are drastically different. For the transition temperature $T \approx 1 \mathrm{~K}$ and $p_{0}=0.5 p_{\mathrm{F}}$, the dimensionless coupling constant $\lambda$ can be estimated as $\lambda \approx 0.27$. The resulting ratios of the spatial scale $\xi_{\star} / \xi=0.022$ and time scale $\tau^{\prime \prime} / \tau^{\prime}=0.028$ are quite small. If we approximate the conductivities by the values $1 / \sigma_{\mathrm{L}}^{\mathrm{e}} \approx 0.3 \mathrm{k} \Omega$ and $1 / \sigma_{\mathrm{L}}^{\mathrm{h}} \approx 0.18 \mathrm{k} \Omega$ measured in the experiment, the order of magnitude for the transverse resistivities can be estimated as $\rho_{\mathrm{H}}^{\mathrm{ee}} \sim 51 \mu \Omega$ and $\rho_{\mathrm{H}}^{\text {he }} \sim 30 \mu \Omega$, which can be probed by state-of-the-art transport experiments.

The ratio of length $\xi_{\star} / \xi$ and time $\tau^{\prime \prime} / \tau^{\prime}$ scales are expected to grow dramatically with coupling strength $\lambda$. According to microscopic theories [23, 47-49], electronhole bilayers experience the crossover from the weak coupling regime to the strong coupling regime as the density of charge carries decreases. In the QW-QW bilayers with the conventional spectrum, the strong coupling regime corresponds to the Bose-Einstein condensate (BEC) of indirect excitons that represent a bound state of electron and hole [66]. In the systems with the gapless Dirac spectrum the strong coupling regime corresponds to the multiband BCS-like paired state [47-49] where pairing correlations also span to remote bands (valence band in the layer with excess of electrons and conduction band in the layer with excess of holes). The strong coupling regime for the hybrid graphene-GaAs bilayers has not been addressed microscopically yet. For the sake of es- timations, we fix the charge carrier concentrations and treat $\lambda$ as an independent parameter. The corresponding dependence of the ratios is presented in Fig. 5. The ratio $\xi_{\star} / \xi$ is much smaller than $\tau^{\prime \prime} / \tau^{\prime}$ due to the small factor $v_{\mathrm{e}} /\left(v_{\mathrm{e}}+v_{\mathrm{h}}\right)$ in front of $\xi_{\star}$. Its small magnitude reflects the fact that the electron velocity is much smaller than the velocity of Dirac holes. As a result, the Cooper pair momentum is mostly carried by the electron, which is free of nontrivial geometries. However, it should be stressed that the calculations presented in Fig. 5 are based on the weak coupling BCS theory with a contact pseudopotential and can be used only for guidance. For $\lambda \approx 0.8$ the ratios of scales are $\xi_{\star} / \xi=0.08$ and $\tau^{\prime \prime} / \tau^{\prime}=0.32$. As a result, the order of magnitude for the transverse resistivities can be estimated as $\rho_{\mathrm{H}}^{\text {ee }} \sim 8.9 \mathrm{~m} \Omega$ and $\rho_{\mathrm{H}}^{\text {he }} \sim 5.3 \mathrm{~m} \Omega$.

The anomalous Hall current in semiconductor QWs requires the symmetry between two valleys in graphene to be broken. The selective formation of fluctuating CPs involving holes only from one of two valleys can be achieved in the presence of a circularly polarized light-induced population imbalance of Dirac holes [67-69]. Due to the exceptional sensitivity of $\mathrm{CP}$ condensation in weakto-moderate coupling regime to the electron-hole imbalance $[57,58]$, even a small asymmetry $\delta \varepsilon_{\mathrm{F}} \sim T_{0}$ is sufficient to suppress correlations with one of two valleys.

The presence of two Dirac cones with opposite chiralities can be avoided in hybrid double-layer systems formed by magnetic topological insulator films (e.g., $\mathrm{MnB}_{2} \mathrm{Te}_{4}$ ) and semiconductor QWs. Recently discovered magnetic topological insulators [70-73] have already been successfully incorporated in different heterostructures, and these hybrid double-layer systems are within present technological capabilities.

In the present work, we have focused on the Aslamazov-Larkin effect, which describes the direct contribution of fluctuating CPs to the conductivity tensor. The Maki-Thompson contribution also exists [3234], originating from the Andreev scattering of electrons and holes for fluctuating CPs. However, the interplay between the nontrivial geometry of fluctuating CPs and Andreev scattering is beyond the scope of the present paper.

The absence of a global gap in the spectrum of the GL Hamiltonian does not permit topologically stable edges or domain wall modes. In other systems with intertwined modes and no global gap (e.g., topological exciton-polaritons [74, 75]), this obstacle is usually overcome by applying an external periodic potential with triagonal (or hexagonal) symmetry. Hexagonal periodic textures of sublattice asymmetry $\delta(\mathbf{r})$ can be easily engineered via a small twist between graphene and its substrate and follow the resulting Moire pattern. However, the physics of fluctuating CPs and the possibility of edge modes in this regime are beyond the scope of the present paper.

In recent years, the Cooper pairing of spin-orbit cou- 
pled fermions has attracted much attention [76, 77]. In particular, research has shown that the nonzero quantum metric of fermionic states result in an additional contribution to the superfluid density and are also manifested in other phenomena [60, 78-81]. Moreover, the additional contribution to the superfluid density is dominant if the fermion dispersion is sufficiently flat. While there are some mathematical connections between these results and the anomalous contribution to paraconductivity $\sigma_{\mathrm{L}}^{\mathrm{G}}$ derived in this paper, there is an essential difference between these phenomena. In our paper, $\sigma_{\mathrm{L}}^{\mathrm{G}}$ is intricately related to the quantum metric characterizing the spectrum of the GL Hamiltonian, but not the spectrum of Bogoliubov quasiparticles in a state with an equilibrium $\mathrm{CP}$ condensate.

Fluctuating CPs formed by electrons and holes are the precursors of their equilibrium condensation. The demonstrated presence of two (almost) degenerate competing channels and the instability toward Cooper pairing with a finite center of mass momentum suggest that the physics of equilibrium hybrid electron-hole condensate are very rich and unconventional. We leave further investigations of this aspect for future work.

To conclude, we have demonstrated that the spectrum of fluctuating electron-hole CPs in a hybrid grapheneGaAs bilayer is topologically nontrivial. Their nontrivial geometries are manifested in anomalous AslamazovLarkin contributions to the longitudinal and transverse conductivities. The contributions exhibit critical behavior and are singular at the transition temperature, and we have discussed possible setups for their experimental observation.

\section{ACKNOWLEDGMENTS}

D. K. E. acknowledges useful discussions with D. Culcer and O. Bleu. This research has been supported by the Australian Research Council Centre of Excellence in Future Low-Energy Electronics Technologies.

* dmitry.efimkin@monash.edu

[1] D. Xiao, M.-C. Chang, and Q. Niu, Berry phase effects on electronic properties, Rev. Mod. Phys. 82, 1959 (2010).

[2] R. Resta, Manifestations of Berry's phase in molecules and condensed matter, Journal of Physics: Condensed Matter 12, R107 (2000).

[3] N. Nagaosa, J. Sinova, S. Onoda, A. H. MacDonald, and N. P. Ong, Anomalous Hall effect, Rev. Mod. Phys. 82, 1539 (2010).

[4] A. P. Schnyder, S. Ryu, A. Furusaki, and A. W. W. Ludwig, Classification of topological insulators and superconductors in three spatial dimensions, Phys. Rev. B 78, 195125 (2008).
[5] M. Z. Hasan and C. L. Kane, Colloquium: Topological insulators, Rev. Mod. Phys. 82, 3045 (2010).

[6] X.-L. Qi and S.-C. Zhang, Topological insulators and superconductors, Rev. Mod. Phys. 83, 1057 (2011).

[7] M. Sato and Y. Ando, Topological superconductors: a review, Reports on Progress in Physics 80, 076501 (2017).

[8] Y. E. Lozovik and V. Yudson, Feasibility of superfluidity of paired spatially separated electrons and holes; a new superconductivity mechanism, JETP Lett. 22, 274 (1975).

[9] Y. E. Lozovik and V. Yudson, A new mechanism for superconductivity: pairing between spatially separated electrons and holes, Sov. Phys. JETP 44, 389 (1976).

[10] S. I. Shevchenko, Theory of superconductivity in the systems with pairing of spatially separated electrons and holes, Sov. J. Low Temp. Phys 2, 251 (1976).

[11] S. Saberi-Pouya, S. Conti, A. Perali, A. F. Croxall, A. R. Hamilton, F. m. c. M. Peeters, and D. Neilson, Experimental conditions for the observation of electron-hole superfluidity in GaAs heterostructures, Phys. Rev. B 101, 140501 (2020).

[12] D. I. Pikulin and T. Hyart, Interplay of Exciton Condensation and the Quantum Spin Hall Effect in $\mathrm{InAs} / \mathrm{GaSb}$ Bilayers, Phys. Rev. Lett. 112, 176403 (2014).

[13] D. V. Fil and S. I. Shevchenko, Electron-hole Superconductivity (Review), Low Temperature Physics 44, 867 (2018), https://doi.org/10.1063/1.5052674.

[14] H. Min, R. Bistritzer, J.-J. Su, and A. H. MacDonald, Room-temperature superfluidity in graphene bilayers, Phys. Rev. B 78, 121401 (2008).

[15] Y. E. Lozovik and A. A. Sokolik, Electron-hole pair condensation in a graphene bilayer, JETP Letters 87, 55 (2008).

[16] C.-H. Zhang and Y. N. Joglekar, Excitonic condensation of massless fermions in graphene bilayers, Phys. Rev. B 77, 233405 (2008).

[17] M. Y. Kharitonov and K. B. Efetov, Electron screening and excitonic condensation in double-layer graphene systems, Phys. Rev. B 78, 241401 (2008).

[18] J. Zhang and E. Rossi, Chiral Superfluid States in Hybrid Graphene Heterostructures, Phys. Rev. Lett. 111, 086804 (2013).

[19] Y. E. Lozovik, S. L. Ogarkov, and A. A. Sokolik, Condensation of electron-hole pairs in a two-layer graphene system: Correlation effects, Phys. Rev. B 86, 045429 (2012).

[20] A. Perali, D. Neilson, and A. R. Hamilton, HighTemperature Superfluidity in Double-Bilayer Graphene, Phys. Rev. Lett. 110, 146803 (2013).

[21] D. Neilson, A. Perali, and A. R. Hamilton, Excitonic superfluidity and screening in electron-hole bilayer systems, Phys. Rev. B 89, 060502 (2014).

[22] J.-J. Su and A. H. MacDonald, Spatially indirect exciton condensate phases in double bilayer graphene, Phys. Rev. B 95, 045416 (2017).

[23] S. Conti, A. Perali, F. M. Peeters, and D. Neilson, Multicomponent Electron-Hole Superfluidity and the BCS$B E C$ Crossover in Double Bilayer Graphene, Phys. Rev. Lett. 119, 257002 (2017).

[24] S. Saberi-Pouya, M. Zarenia, A. Perali, T. Vazifehshenas, and F. M. Peeters, High-temperature electron-hole superfluidity with strong anisotropic gaps in double phosphorene monolayers, Phys. Rev. B 97, 174503 (2018).

[25] B. Debnath, Y. Barlas, D. Wickramaratne, M. R. Neupane, and R. K. Lake, Exciton condensate in bilayer transition metal dichalcogenides: Strong coupling regime, 
Phys. Rev. B 96, 174504 (2017).

[26] X. Hu, T. Hyart, D. I. Pikulin, and E. Rossi, Quantummetric-enabled exciton condensate in double twisted bilayer graphene (2020), arXiv:2008.03241 [cond-mat.meshall].

[27] B. Seradjeh, J. E. Moore, and M. Franz, Exciton Condensation and Charge Fractionalization in a Topological Insulator Film, Phys. Rev. Lett. 103, 066402 (2009).

[28] D. K. Efimkin, Y. E. Lozovik, and A. A. Sokolik, Electron-hole pairing in a topological insulator thin film, Phys. Rev. B 86, 115436 (2012).

[29] It should be noted, that these CPs are formed not by electrons, but by spatially separated electrons and holes.

[30] D. K. Efimkin and Y. E. Lozovik, Fluctuational internal Josephson effect in a topological insulator film, Phys. Rev. B 88, 085414 (2013).

[31] D. K. Efimkin, G. W. Burg, E. Tutuc, and A. H. MacDonald, Tunneling and fluctuating electron-hole Cooper pairs in double bilayer graphene, Phys. Rev. B 101, 035413 (2020).

[32] B. Y.-K. Hu, Prospecting for the Superfluid Transition in Electron-Hole Coupled Quantum Wells Using Coulomb Drag, Phys. Rev. Lett. 85, 820 (2000).

[33] M. P. Mink, H. T. C. Stoof, R. A. Duine, M. Polini, and G. Vignale, Probing the Topological Exciton Condensate via Coulomb Drag, Phys. Rev. Lett. 108, 186402 (2012).

[34] M. P. Mink, H. T. C. Stoof, R. A. Duine, M. Polini, and G. Vignale, Unified Boltzmann transport theory for the drag resistivity close to an interlayer-interaction-driven second-order phase transition, Phys. Rev. B 88, 235311 (2013).

[35] D. K. Efimkin and Y. E. Lozovik, Drag effect and Cooper electron-hole pair fluctuations in a topological insulator film, Phys. Rev. B 88, 235420 (2013).

[36] A. A. Varlamov, A. Galda, and A. Glatz, Fluctuation spectroscopy: From Rayleigh-Jeans waves to Abrikosov vortex clusters, Rev. Mod. Phys. 90, 015009 (2018).

[37] W. J. Skocpol and M. Tinkham, Fluctuations near superconducting phase transitions, Reports on Progress in Physics 38, 1049 (1975).

[38] I. Larkin and A. Valralmov, Theory of Fluctuations in Superconductors (Clarendon, Oxford, 2005).

[39] L. G. Aslamazov and L. A.I., Fluctuation-induced magnetic susceptibility of superconductors and normal metals, Sov. Phys. JETP 40, 321 (1975).

[40] L. Aslamasov and A. Larkin, The influence of fluctuation pairing of electrons on the conductivity of normal metal, Physics Letters A 26, 238 (1968).

[41] G. W. Burg, N. Prasad, K. Kim, T. Taniguchi, K. Watanabe, A. H. MacDonald, L. F. Register, and E. Tutuc, Strongly Enhanced Tunneling at Total Charge Neutrality in Double-Bilayer Graphene-WSe 2 Heterostructures, Phys. Rev. Lett. 120, 177702 (2018).

[42] Z. Wang, D. A. Rhodes, K. Watanabe, T. Taniguchi, J. C. Hone, J. Shan, and K. F. Mak, Evidence of hightemperature exciton condensation in two-dimensional atomic double layers, Nature 574, 76 (2019).

[43] C. P. Morath, J. A. Seamons, J. L. Reno, and M. P. Lilly, Density imbalance effect on the Coulomb drag upturn in an undoped electron-hole bilayer, Phys. Rev. B 79, 041305 (2009).

[44] A. F. Croxall, K. Das Gupta, C. A. Nicoll, M. Thangaraj, H. E. Beere, I. Farrer, D. A. Ritchie, and M. Pepper, Anomalous Coulomb Drag in Electron-Hole Bilayers,
Phys. Rev. Lett. 101, 246801 (2008).

[45] A. F. Croxall, K. Das Gupta, C. A. Nicoll, H. E. Beere, I. Farrer, D. A. Ritchie, and M. Pepper, Possible effect of collective modes in zero magnetic field transport in an electron-hole bilayer, Phys. Rev. B 80, 125323 (2009).

[46] A. Gamucci, D. Spirito, M. Carrega, B. Karmakar, A. Lombardo, M. Bruna, L. N. Pfeiffer, K. W. West, A. C. Ferrari, M. Polini, and V. Pellegrini, Anomalous low-temperature Coulomb drag in graphene-GaAs heterostructures, Nature Communications 5, 5824 (2014).

[47] I. Sodemann, D. A. Pesin, and A. H. MacDonald, Interaction-enhanced coherence between two-dimensional Dirac layers, Phys. Rev. B 85, 195136 (2012).

[48] Y. E. Lozovik and A. A. Sokolik, Ultrarelativistic electron-hole pairing in graphene bilayer, The European Physical Journal B 73, 195 (2010).

[49] S. Conti, A. Perali, F. M. Peeters, and D. Neilson, Multicomponent screening and superfluidity in gapped electronhole double bilayer graphene with realistic bands, Phys. Rev. B 99, 144517 (2019).

[50] These arguments are valid in the presence of substrate induced gap if is magnitude is small.

[51] It should be noted that after the formal electron-hole transformation the connections to the BCS theory of superconductivity established in early works on electronhole Cooper pair condensation [8-10] become apparent.

[52] The presence of two (almost) degenerate competing channels also occurs for other hybrid double-layer systems, including bilayer graphene-GaAs and monolayer graphenebilayer graphene.

[53] In a graphene/graphene bilayer, the angle factor must be modified as $\Lambda_{\mathbf{p p}^{\prime}} \rightarrow \Lambda_{\mathbf{p p}^{\prime}}^{2}$ for intravalley CPs and as $\Lambda_{\mathbf{p p}^{\prime}} \rightarrow\left|\Lambda_{\mathbf{p p}^{\prime}}\right|^{2}$ for intervalley CPs. In the absence of sublattice asymmetry, $\delta=0$, the modified angle factor is the same, $\Lambda_{\mathbf{p p}^{\prime}}=\cos ^{2}\left(\phi_{\mathbf{p}, \mathbf{p}^{\prime}} / 2\right)$.

[54] It is instructive to rescale the fields as $\Delta^{\mathrm{A}} \rightarrow \Delta^{\mathrm{A}} / \sqrt{\nu_{0}} s_{\mathrm{F}}$ and $\Delta^{\mathrm{B}} \rightarrow \Delta^{\mathrm{B}} / \sqrt{\nu_{0}} c_{\mathrm{F}}$. See Appendix A for detailed derivations.

[55] P. Fulde and R. A. Ferrell, Superconductivity in a Strong Spin-Exchange Field, Phys. Rev. 135, A550 (1964).

[56] I. Larkin and Y. Ovchinikov, Nonuniform state of superconductors, Sov. Phys. JETP 20, 762 (1965).

[57] B. Seradjeh, Topological exciton condensate of imbalanced electrons and holes, Phys. Rev. B 85, 235146 (2012).

[58] D. K. Efimkin and Y. Lozovik, Electron-hole pairing with nonzero momentum in a graphene bilayer, JETP 113, 880 (2011).

[59] D. N. Page, Geometrical description of Berry's phase, Phys. Rev. A 36, 3479 (1987).

[60] E. Rossi, Quantum metric and correlated states in twodimensional systems, Current Opinion in Solid State and Materials Science 25, 100952 (2021).

[61] O. Bleu, D. D. Solnyshkov, and G. Malpuech, Measuring the quantum geometric tensor in two-dimensional photonic and exciton-polariton systems, Phys. Rev. B 97, 195422 (2018).

[62] N. Nagaosa, J. Sinova, S. Onoda, A. H. MacDonald, and N. P. Ong, Anomalous Hall effect, Rev. Mod. Phys. 82, 1539 (2010).

[63] M. Borunda, T. S. Nunner, T. Lück, N. A. Sinitsyn, C. Timm, J. Wunderlich, T. Jungwirth, A. H. MacDonald, and J. Sinova, Absence of Skew Scattering in TwoDimensional Systems: Testing the Origins of the Anoma- 
lous Hall Effect, Phys. Rev. Lett. 99, 066604 (2007).

[64] D. Culcer, A. Sekine, and A. H. MacDonald, Interband coherence response to electric fields in crystals: Berryphase contributions and disorder effects, Phys. Rev. B 96, 035106 (2017).

[65] It should be noted that a valley current is induced and can be detected via nonlocal measurements [82, 83].

[66] P. López Ríos, A. Perali, R. J. Needs, and D. Neilson, Evidence from Quantum Monte Carlo Simulations of LargeGap Superfluidity and BCS-BEC Crossover in Double Electron-Hole Layers, Phys. Rev. Lett. 120, 177701 (2018).

[67] D. S. L. Abergel and T. Chakraborty, Generation of valley polarized current in bilayer graphene, Applied Physics Letters 95, 062107 (2009), https://doi.org/10.1063/1.3205117.

[68] W. Yao, D. Xiao, and Q. Niu, Valley-dependent optoelectronics from inversion symmetry breaking, Phys. Rev. B 77, 235406 (2008).

[69] T. Farajollahpour and A. Phirouznia, The role of the strain induced population imbalance in Valley polarization of graphene: Berry curvature perspective, Scientific Reports 7, 17878 (2017).

[70] C. Liu, Y. Wang, H. Li, Y. Wu, Y. Li, J. Li, K. He, $\mathrm{Y}$. Xu, J. Zhang, and Y. Wang, Robust axion insulator and Chern insulator phases in a two-dimensional antiferromagnetic topological insulator, Nature Materials 19, $522(2020)$.

[71] Y. L. Chen, J.-H. Chu, J. G. Analytis, Z. K. Liu, K. Igarashi, H.-H. Kuo, X. L. Qi, S. K. Mo, R. G. Moore, D. H. Lu, M. Hashimoto, T. Sasagawa, S. C. Zhang, I. R. Fisher, Z. Hussain, and Z. X. Shen, Massive Dirac Fermion on the Surface of a Magnetically Doped Topological Insulator, Science 329, 659 (2010).

[72] Y. Deng, Y. Yu, M. Z. Shi, Z. Guo, Z. Xu, J. Wang, X. H. Chen, and Y. Zhang, Quantum anomalous Hall effect in intrinsic magnetic topological insulator MnBi2Te4, Science 367, 895 (2020).

[73] C. X. Trang, Q. Li, Y. Yin, J. Hwang, G. Akhgar, I. Di Bernardo, A. Grubišić-Čabo, A. Tadich, M. S. Fuhrer, S.-K. Mo, N. V. Medhekar, and M. T. Edmonds, Crossover from 2D Ferromagnetic Insulator to Wide Band Gap Quantum Anomalous Hall Insulator in Ultrathin MnBi2Te4, ACS Nano 15, 13444 (2021).

[74] T. Karzig, C.-E. Bardyn, N. H. Lindner, and G. Refael, Topological Polaritons, Phys. Rev. X 5, 031001 (2015).

[75] A. V. Nalitov, D. D. Solnyshkov, and G. Malpuech, Polariton $\mathbb{Z}$ Topological Insulator, Phys. Rev. Lett. 114, 116401 (2015).

[76] J. J. Kinnunen, J. E. Baarsma, J.-P. Martikainen, and P. Torma, The Fulde-Ferrell-Larkin-Ovchinnikov state for ultracold fermions in lattice and harmonic potentials: a review, Reports on Progress in Physics 81, 046401 (2018).

[77] K. Gubbels and H. Stoof, Physics Reports 525, 255 (2013), imbalanced Fermi Gases at Unitarity.

[78] S. Peotta and P. Törmä, Superfluidity in topologically nontrivial flat bands, Nature Communications 6, 8944 (2015).

[79] L. Liang, T. I. Vanhala, S. Peotta, T. Siro, A. Harju, and P. Törmä, Band geometry, Berry curvature, and superfluid weight, Phys. Rev. B 95, 024515 (2017).

[80] Z. Wang, G. Chaudhary, Q. Chen, and K. Levin, Quantum geometric contributions to the BKT transition: Be- yond mean field theory, Phys. Rev. B 102, 184504 (2020).

[81] M. Iskin, Quantum-metric contribution to the pair mass in spin-orbit-coupled Fermi superfluids, Phys. Rev. A 97, 033625 (2018).

[82] Y. Shimazaki, M. Yamamoto, I. V. Borzenets, K. Watanabe, T. Taniguchi, and S. Tarucha, Generation and detection of pure valley current by electrically induced Berry curvature in bilayer graphene, Nature Physics 11, 1032 (2015).

[83] J. R. Schaibley, H. Yu, G. Clark, P. Rivera, J. S. Ross, K. L. Seyler, W. Yao, and X. Xu, Valleytronics in $2 D$ materials, Nature Reviews Materials 1, 16055 (2016).

[84] C. H. Wong and Y. Tserkovnyak, Quantum kinetic equation in phase-space textured multiband systems, Phys. Rev. B 84, 115209 (2011). 


\section{APPENDIX A. FIELD THEORETICAL APPROACH FOR FLUCTUATING COOPER PAIRS}

\section{A1. The model}

This Appendix presents the quantum field theory approach to describe fluctuating Cooper pairs (CPs) in the hybrid graphene/quantum well bilayer. Following the reported experimental setup [46], we assume the presence of excess electrons in the quantum well (QW) and their deficit in graphene. The electrons can be described by a quantum field $e_{\tau \mathbf{r}}$ and their thermodynamic action represents an integral over the imaginary Matsubara time $\tau$ and is given by

$$
S_{\mathrm{e}}=\int_{0}^{\beta} \int d \mathbf{r} e_{\tau \mathbf{r}}^{\dagger}\left(\partial_{\tau}+\frac{\mathbf{p}^{2}-p_{\mathrm{F}}^{2}}{2 m_{\mathrm{e}}}\right) e_{\tau \mathbf{r}} .
$$

Electrons have the conventional quadratic dispersion with the mass $m_{\mathrm{e}}$ and $p_{\mathrm{F}}$ is their Fermi momentum. In the weak-to-moderate coupling regime the electron-hole correlations span only in the vicinity of the Fermi level. As a result, the spectrum of electrons can be linearized and their Green function can be approximated as

$$
G^{\mathrm{e}}\left(i \omega_{n}, \mathbf{p}\right)=\frac{1}{i \omega_{n}-\epsilon_{\mathbf{p}}^{\mathrm{e}}}, \quad \epsilon_{\mathrm{p}}^{\mathrm{e}}=v_{\mathrm{e}}\left(p-p_{\mathrm{F}}\right), \quad v_{\mathrm{e}}=p_{\mathrm{F}} / m_{\mathrm{h}} .
$$

Here $\omega_{n}=(2 n+1) \pi T$ is the fermionic Matsubara frequency. The low-energy electronic states in graphene are concentrated near two inequivalent valleys $\left(\mathrm{K}\right.$ and $\left.\mathrm{K}^{\prime}\right)$, which are labeled by the index $\zeta= \pm 1$. They are described by the spinor field operator $h_{\mathbf{r}}=\left(h_{\mathbf{r}}^{\mathrm{A}}, h_{\mathbf{r}}^{\mathrm{B}}\right)$ and their pseudospin corresponds to the the sublattice (A and B) degree of freedom of the honeycomb lattice. We will assume the deficit of electrons in graphene and refer the valence band states as holes, however it is instructive not to perform the transformation to field operators of holes. As a result, the corresponding contribution to the action is given by

$$
S_{\mathrm{h}}=\int_{0}^{\beta} \int d \mathbf{r}\left[h_{\tau \mathbf{r}}^{\dagger}\left(\partial_{\tau}+v\left(\zeta p_{x} \sigma_{x}+p_{y} \sigma_{y}\right)+\delta \sigma_{z}+\epsilon_{\mathrm{F}}^{\mathrm{h}}\right) h_{\tau \mathbf{r}}\right] .
$$

Here $v$ is their velocity and $\epsilon_{\mathrm{F}}^{\mathrm{h}}$ is the Fermi energy. The small energy asymmetry between sublattices $\delta \ll \epsilon_{\mathrm{F}}^{\mathrm{h}}$ can be induced by the substrate engineering. The empty conduction band in graphene can be just truncated, while the energy and the spinor wave function for the valence band electrons are given by

$$
\epsilon_{\mathbf{p}}^{\mathrm{h}}=-\sqrt{(v p)^{2}+\delta^{2}}, \quad|\mathbf{p}\rangle_{\mathrm{h}}=\left(\begin{array}{c}
\sin \left(\theta_{\mathbf{p}} / 2\right) e^{-i \zeta \phi_{\mathbf{p}} / 2} \\
-\zeta \cos \left(\theta_{\mathbf{p}} / 2\right) e^{i \zeta \phi_{\mathbf{p}} / 2}
\end{array}\right)
$$

Here $\phi_{\mathbf{p}}$ is the polar angle for vector $\mathbf{p}$ and $\cos \left(\theta_{\mathbf{p}}\right)=\delta / \epsilon_{\mathbf{p}}$. The chirality of the pseudospin-momentum coupling for Dirac electrons is valley dependent but not their dispersion. We further assume that the Fermi momentum of electrons matches with the one $p_{\mathrm{F}}$ for holes, that is the most favorable regime for their Cooper pairing. As a result, the Green function for holes can be approximated as

$$
\hat{G}^{\mathrm{h}}\left(i \omega_{n}, \mathbf{p}\right)=\frac{|\mathbf{p}\rangle_{\mathrm{h} \mathrm{h}}\langle\mathbf{p}|}{i \omega_{n}-\epsilon_{\mathbf{p}}^{\mathrm{h}}}, \quad \epsilon_{\mathbf{p}}^{\mathrm{h}}=v_{\mathrm{h}}\left(p-p_{\mathrm{F}}\right), \quad v_{\mathrm{h}}=v^{2} p_{\mathrm{F}} / \epsilon_{\mathrm{F}}^{\mathrm{h}}
$$

Here we have linearized the spectrum of Dirac holes and the matrix $|\mathbf{p}\rangle_{\mathrm{h} \mathrm{h}}\langle\mathbf{p}|$ is given by

$$
|\mathbf{p}\rangle_{\mathrm{h} \mathrm{h}}\langle\mathbf{p}|=\left(\begin{array}{cc}
\sin ^{2}\left(\theta_{\mathbf{p}} / 2\right) & -\frac{\zeta}{2} \sin \left(\theta_{\mathbf{p}}\right) e^{-i \zeta \phi_{\mathbf{p}}} \\
-\frac{\zeta}{2} \sin \left(\theta_{\mathbf{p}}\right) e^{i \zeta \phi_{\mathbf{p}}} & \cos ^{2}\left(\theta_{\mathbf{p}} / 2\right)
\end{array}\right)=\frac{1}{2 \epsilon_{\mathbf{p}}^{\mathrm{h}}}\left(\begin{array}{cc}
\epsilon_{\mathbf{p}}^{\mathrm{h}}-\delta & -v\left(\zeta p_{x}-i p_{y}\right) \\
-v\left(\zeta p_{x}-i p_{y}\right) & \epsilon_{\mathbf{p}}^{\mathrm{h}}+\delta
\end{array}\right)
$$

The phase intertwining between sublattices is the signature of the nontrivial band geometries for Dirac holes, which has been discussed in the main text and is passed on to the fluctuating CPs. Fluctuating CPs are formed due to the attractive Coulomb interactions between electrons and holes. Being effectively screened, the interactions can be approximated by the contact pseudopotential with momentum-independent Fourier transform $V$. We also neglect the scattering between two valleys since they are well separated in momentum space. The resulting contribution to the action is given by

$$
S_{\mathrm{int}}=\int_{0}^{\beta} \int d \mathbf{r} V e_{\tau \mathbf{r}}^{\dagger} h_{\tau \mathbf{r}}^{\dagger} h_{\tau \mathbf{r}} e_{\tau \mathbf{r}} \rightarrow \int_{0}^{\beta} \int d \mathbf{r}\left[\frac{1}{V} \Delta_{\tau \mathbf{r}}^{\dagger} \Delta_{\tau \mathbf{r}}+e_{\tau \mathbf{r}}^{\dagger} \Delta_{\tau \mathbf{r}} h_{\tau \mathbf{r}}+h_{\tau \mathbf{r}}^{\dagger} \Delta_{\tau \mathbf{r}}^{\dagger} e_{\tau \mathbf{r}}\right]
$$

Here we have performed the Hubbard-Stratonovich transformation. It eliminates interactions, but introduces the bosonic field $\Delta_{\tau \mathbf{r}}=\left\{\Delta_{\tau \mathbf{r}}^{\mathrm{A}}, \Delta_{\tau \mathbf{r}}^{\mathrm{B}}\right\}$, which describes the selective Cooper pairing with Dirac holes only at one of two sublattices (A and B). The action of the model $S=S_{\mathrm{e}}+S_{\mathrm{h}}+S_{\mathrm{int}}$ is the starting point for the derivation of fluctuating CPs dynamics, which is presented in the next subsection. 


\section{A2. Fluctuating Cooper pairs}

We further assume that the system is above the transition temperature. As a result there is no equilibrium Cooper pair condensate $\langle\Delta\rangle=0$, but there are its Gaussian fluctuations, which are usually interpreted as fluctuating CPs. Integrating fermions out and expanding the resulting action up to the quadratic order in $\Delta_{\tau \mathbf{r}}$ results in

$$
S_{\Delta}=T \sum_{p_{n} \mathbf{q}} \bar{\Delta}_{p_{n} \mathbf{q}}\left(\frac{1}{V}-\hat{\Pi}\left(i p_{n}, \mathbf{q}\right)\right) \Delta_{p_{n} \mathbf{q}}
$$

Here $p_{n}=2 \pi n T$ is the bosonic Matsubara frequency. $\hat{\Pi}\left(i p_{n}, \mathbf{q}\right)$ is the single step pair propagator in the Cooper ladder sum and is given by

$$
\hat{\Pi}\left(i p_{n}, \mathbf{q}\right)=T \sum_{\omega_{n}, \mathbf{p}} \hat{G}^{\mathrm{e}}\left(i \omega_{n}, \mathbf{p}\right) G^{\mathrm{h}}\left(i \omega_{n}-i p_{n}, \mathbf{p}-\mathbf{q}\right)=-\sum_{\mathbf{p}}|\mathbf{p}\rangle_{\mathrm{e}} \mathrm{e}\langle\mathbf{p}| \frac{\tanh \left(\frac{\xi_{\mathbf{p}}^{\mathrm{e}}}{2 T}\right)+\tanh \left(\frac{\xi_{\mathbf{p}-\mathbf{q}}^{\mathrm{h}}}{2 T}\right)}{2\left(i p_{n}-\xi_{\mathbf{p}}^{\mathrm{e}}-\xi_{\mathbf{p}-\mathbf{q}}^{\mathrm{h}}\right)} .
$$

It is instructive to shift the momenta $\mathbf{p}$ and $\mathbf{p}-\mathbf{q}$ as follows

$$
\begin{aligned}
\mathbf{p} \rightarrow \mathbf{p}+\alpha_{\mathrm{e}} \mathbf{q}, & \alpha_{\mathrm{e}}=\frac{v_{h}}{v_{e}+v_{h}}, \\
\mathbf{p}-\mathbf{q} \rightarrow \mathbf{p}-\alpha_{\mathrm{h}} \mathbf{q}, & \alpha_{\mathrm{h}}=\frac{v_{e}}{v_{e}+v_{h}} .
\end{aligned}
$$

The momenta $\mathbf{q}$ is the total momentum for fluctuating CPs and $\mathbf{p}$ can be interpreted as their relative one. The dispersion relations in Eq. (41) are modified as

$$
\xi_{\mathbf{p}+\alpha_{\mathrm{e}} \mathbf{q}}^{\mathrm{e}} \approx \xi_{\mathbf{p}}^{\mathrm{e}}+\frac{v_{*} \mathbf{q} \mathbf{p}}{2 p}, \quad \xi_{\mathbf{p}-\alpha_{\mathrm{h}} \mathbf{q}}^{\mathrm{h}} \approx \xi_{\mathbf{p}}^{\mathrm{h}}-\frac{v_{*} \mathbf{q} \mathbf{p}}{2 p}, \quad \xi_{\mathbf{p}+\alpha_{\mathrm{e}} \mathbf{q}}^{\mathrm{e}}+\xi_{\mathbf{p}-\alpha_{\mathrm{h}} \mathbf{q}}^{\mathrm{h}} \approx 2 v_{0}\left(p-p_{\mathrm{F}}\right) .
$$

Here we have introduced $v_{0}=\left(v_{e}+v_{h}\right) / 2$ and $v_{*}=2 v_{\mathrm{e}} v_{\mathrm{h}} /\left(v_{\mathrm{e}}+v_{\mathrm{h}}\right)$. After the momentum shift, Eq.(42), the denominator in Eq. (41) becomes independent on momentum $\mathbf{q}$. As a result, the expansion of $\hat{\Pi}\left(i p_{n}, \mathbf{q}\right)$ in $p_{n}$ and $\mathbf{q}$ becomes straightforward and results in

$$
\hat{\Pi}\left(i p_{n}, \mathbf{q}\right)=\left(\begin{array}{cc}
s_{\mathrm{F}}^{2} \Pi_{0}\left(i p_{n}, \mathbf{q}\right) & \zeta c_{\mathrm{F}} s_{\mathrm{F}} \frac{\alpha_{e} q e^{-i \zeta \phi_{\mathbf{q}}}}{4 p_{\mathrm{F}}} \Pi_{0}(0,0) \\
\zeta c_{\mathrm{F}} s_{\mathrm{F}} \frac{\alpha_{e} q e^{-i \zeta \phi_{\mathbf{q}}}}{4 p_{\mathrm{F}}} \Pi_{0}(0,0) & \left.c_{\mathrm{F}}^{2} \Pi_{0}\left(i p_{n}, \mathbf{q}\right)\right)
\end{array}\right)
$$

The factors

$$
c_{\mathrm{F}}^{2}=\frac{1}{2}\left(1+\frac{\delta}{\epsilon_{\mathrm{F}}}\right), \quad s_{\mathrm{F}}^{2}=\frac{1}{2}\left(1-\frac{\delta}{\epsilon_{\mathrm{F}}}\right) .
$$

give the probability to find the Fermi level hole at sublattice A or B, respectively. $\Pi_{0}\left(i p_{n}\right.$, q) can be interpreted as a single step pair propagator in the Cooper ladder sum of a bilayer system without the pseudospin degree of freedom and is given by

$$
\Pi_{0}\left(i p_{n}, \mathbf{q}\right)=\nu_{0}\left[\ln \left(\frac{2 e^{C} \epsilon_{\mathrm{C}}}{\pi T}\right)-\left|p_{n}\right| \tau^{\prime}-i p_{n} \tau^{\prime \prime}-\xi^{2} \mathbf{q}^{2}\right], \quad \tau^{\prime}=\frac{\pi}{8 T}, \quad \tau^{\prime \prime}=\frac{\ln \left(\frac{2 e^{C} \epsilon_{\mathrm{c}}}{\pi T}\right)}{2 v_{0} p_{\mathrm{F}}}, \quad \xi^{2}=\frac{7 \Xi(3) v_{*}^{2}}{32 \pi^{2} T^{2}}
$$

Here $\epsilon_{0}=\sqrt{v_{\mathrm{e}} v_{\mathrm{h}}} p_{0}$ is determined by the momentum cutoff $p_{0} . C=0.577$ is the Euiler constant, $\Xi(x)$ is the Zeta function and $\nu_{0}=p_{\mathrm{F}} / 2 \pi \hbar^{2} v_{0}$ is the density of states defined respect to the average Fermi velocity of electrons and holes. If we rescale the bosonic fields as $\Delta^{\mathrm{A}} \rightarrow \Delta^{\mathrm{A}} / \sqrt{\nu_{0}} s_{\mathrm{F}}$ and $\Delta^{\mathrm{B}} \rightarrow \Delta^{\mathrm{B}} / \sqrt{\nu_{0}} c_{\mathrm{F}}$, the action describing fluctuating CPs, Eq. $40 S_{\Delta}$, simplifies as

$$
S_{\Delta}=T \sum_{p_{n} \mathbf{q}} \bar{\Delta}_{p_{n} \mathbf{q}} \hat{L}^{-1}\left(i p_{n}, \mathbf{q}\right) \Delta_{p_{n} \mathbf{q}}
$$

where $\hat{L}^{-1}\left(i p_{n}, \mathbf{q}\right)$ is the inverse dimensionless CP propagator given by

$$
\hat{L}^{-1}\left(i p_{n}, \mathbf{q}\right)=\left|p_{n}\right| \tau^{\prime}-i p_{n} \tau^{\prime \prime}+\hat{H}_{\mathrm{GL}}, \quad \hat{H}_{\mathrm{GL}}=\left(\begin{array}{cc}
\varepsilon_{\mathrm{A}}+\xi^{2} \mathbf{q}^{2} & -\xi_{\star}\left(\zeta q_{x}-i q_{y}\right) \\
-\xi_{\star}\left(\zeta q_{x}-i q_{y}\right) & \varepsilon_{\mathrm{B}}+\xi^{2} \mathbf{q}^{2}
\end{array}\right) .
$$


The Hermitian matrix $\hat{H}_{\mathrm{GL}}$ can be referred to as the Ginzburg-Landau Hamiltonian. It describes two dissipative bosonic modes $\left(\Delta_{\mathrm{A}}\right.$ and $\left.\Delta_{\mathrm{A}}\right)$ that are intertwined by the effective pseudospin-orbit interactions with strength parametrized by the length $\xi_{\star}=\alpha_{\mathrm{e}} \ln \left(2 e^{C} \epsilon_{\mathrm{c}} / \pi T\right) / 4 p_{\mathrm{F}}$. Each mode has a temperature-dependent energy gap

$$
\epsilon_{\mathrm{A}(\mathrm{B})}=\ln \left[\frac{T}{T_{\mathrm{A}(\mathrm{B})}}\right], \quad T_{\mathrm{A}(\mathrm{B})}=\frac{2 e^{C} e^{-\frac{1}{\lambda_{\mathrm{A}(\mathrm{B})}}}}{\pi}, \quad \lambda_{\mathrm{A}}=s_{\mathrm{F}}^{2} \nu_{0} V, \quad \lambda_{\mathrm{B}}=c_{\mathrm{F}}^{2} \nu_{0} V .
$$

The gap vanishes at the transition temperature $\varepsilon_{\mathrm{A}(\mathrm{B})}=\ln \left[T / T_{\mathrm{A}(\mathrm{B})}^{0}\right]$ of the Cooper pair condensation with zero center of mass momentum. It should be mentioned that coefficients $\tau^{\prime \prime}$ and $\xi_{\star}$ can be rewritten as $\tau^{\prime \prime}=1 / 2 \lambda v_{0} p_{\mathrm{F}}$ and $\xi_{\star}=1 / 4 \lambda p_{\mathrm{F}}$ where $\lambda=\max \left[\lambda_{\mathrm{A}}, \lambda_{\mathrm{B}}\right]$ is the coupling constant of the leading channel.

\section{A3. The spectrum of Ginzburg-Landau Hamiltonian}

Eigenvalues for $\hat{H}_{\mathrm{GL}}$ are labeled by $\gamma= \pm$ and are given by

$$
\varepsilon_{\gamma \mathbf{q}}=\varepsilon_{\mathrm{s}}+\xi^{2} \mathbf{q}^{2}+\gamma d_{\mathbf{q}}, \quad d_{\mathbf{q}}=\sqrt{\varepsilon_{\mathrm{z}}^{2}+\xi_{\star}^{2} \mathbf{q}^{2}}, \quad \epsilon_{\mathrm{s}}=\frac{1}{2}\left(\epsilon_{\mathrm{A}}+\epsilon_{\mathrm{B}}\right), \quad \epsilon_{\mathrm{z}}=\frac{1}{2}\left(\epsilon_{\mathrm{A}}-\epsilon_{\mathrm{B}}\right) .
$$

They can be interpreted as energies for fluctuating CPs eigenmodes and their dispersion is discussed in detail in the main part of the paper. The fluctuating CPs with finite center of mass momentum q represent a superposition of $\Delta_{\mathrm{A}}$ and $\Delta_{\mathrm{B}}$ as

$$
|+, \mathbf{q}\rangle_{\mathrm{C}}=\left(\begin{array}{c}
\cos \left(\vartheta_{\mathbf{q}} / 2\right) \\
-\zeta \sin \left(\theta_{\mathbf{q}} / 2\right) e^{i \zeta \phi_{\mathbf{q}}}
\end{array}\right), \quad|-, \mathbf{q}\rangle_{\mathrm{C}}=\left(\begin{array}{c}
\zeta \sin \left(\vartheta_{\mathbf{q}} / 2\right) e^{-i \zeta \phi_{\mathbf{q}}} \\
\cos \left(\vartheta_{\mathbf{q}} / 2\right)
\end{array}\right), \quad \cos \left(\vartheta_{\mathbf{q}}\right)=\frac{\epsilon_{\mathrm{z}}}{\sqrt{\epsilon_{\mathrm{z}}^{2}+\xi_{\star}^{2} \mathbf{q}^{2}}}
$$

They are intertwined with the valley dependent phase factor $e^{-i \zeta \phi_{\mathbf{q}}}$, which reflects nontrivial geometries for fluctuating CPs.

The derived expressions, Eqs. (50) and (51), allow to perform the spectral decomposition of CP propagator $L\left(i p_{n}, \mathbf{q}\right)$ and its retarded (advanced) $L^{\mathrm{R}(A)}(\omega, \mathbf{q})$ counterparts as

$$
\hat{L}\left(i p_{n}, \mathbf{q}\right)=\sum_{\gamma} \frac{|\gamma, \mathbf{q}\rangle_{\mathrm{C} C}\langle\gamma, \mathbf{q}|}{\tau^{\prime}\left|p_{n}\right|-i \tau^{\prime \prime} p_{n}+\varepsilon_{\gamma \mathbf{q}}}, \quad \hat{L}^{\mathrm{R}(\mathrm{A})}(\omega, \mathbf{q})=\sum_{\gamma} \frac{|\gamma, \mathbf{q}\rangle_{\mathrm{C} C}\langle\gamma, \mathbf{q}|}{\mp i \omega \tau^{\prime}-\omega \tau^{\prime \prime}+\varepsilon_{\gamma \mathbf{q}}} .
$$

These helpful relations will be used routinely in Appendices B and C.

Due to their dissipative nature, fluctuating CPs cannot be interpreted as bosonic quasiparticles, but are instead overdamped bosonic modes. However, $\hat{H}_{\mathrm{GL}}$ is the Hermitian matrix and its eigenmodes $|\gamma \mathbf{q}\rangle_{\mathrm{C}}$ form a complete basis. $\hat{H}_{\mathrm{GL}}$ can be diagonalized via the unitary transformation governed by the matrix $\hat{U}_{\mathbf{q}}$ given by

$$
\hat{E}_{\mathrm{GL}}(\mathbf{q})=U_{\mathbf{q}} \hat{H}_{\mathrm{GL}}(\mathbf{q}) U_{\mathbf{q}}^{\dagger}, \quad \hat{E}_{\mathrm{GL}}=\left(\begin{array}{cc}
\varepsilon_{+, \mathbf{q}} & 0 \\
0 & \varepsilon_{-, \mathbf{q}}
\end{array}\right), \quad U_{\mathbf{q}}=\left(\begin{array}{cc}
\cos \left(\vartheta_{\mathbf{q}} / 2\right) & \zeta \sin \left(\vartheta_{\mathbf{q}} / 2\right) e^{-i \zeta \phi_{\mathbf{q}}} \\
-\zeta \sin \left(\theta_{\mathbf{q}} / 2\right) e^{i \zeta \phi_{\mathbf{q}}} & \cos \left(\vartheta_{\mathbf{q}} / 2\right) .
\end{array}\right)
$$

This transformation is used in Appendix C, where a derivation of the kinetic equation for fluctuating CPs is presented.

\section{APPENDIX B. LINEAR RESPONSE THEORY AND PARACONDUCTIVITY}

\section{B1. General expression for the conductivity tensor}

This section presents a general expressions for the conductivity tensor, which is derived with the help of the linear response approach. The external electric fields in electron and hole layers that we have disregarded so far can be described by position independent vector potentials $\mathbf{a}_{t}^{\mathrm{e}}$ and $\mathbf{a}_{t}^{\mathrm{h}}$. Components of a fluctuating CP are spatially separated and have the opposite charge. That is why the Peierls substitution introduces the vector potential to the Ginzburg-Landau Hamiltonian as

$$
\hat{H}_{\mathrm{GL}}(\mathbf{q}) \rightarrow \hat{H}_{\mathrm{GL}}\left(\mathbf{q}-\frac{e}{c} \mathbf{a}_{t}^{\mathrm{eh}}\right), \quad \mathbf{a}_{t}^{\mathrm{eh}}=\mathbf{a}_{t}^{\mathrm{e}}-\mathbf{a}_{t}^{\mathrm{h}} .
$$


Fluctuating CPs are affected by the electric field difference across the bilayer and their motion results in electric currents in both layers. These currents have the same magnitude but the opposite signs. Really, the current operators for two layers have the opposite signs as

$$
\mathbf{J}^{\mathrm{e}(\mathrm{h})}(\mathbf{q})= \pm e \mathbf{v}(\mathbf{q}), \quad \mathbf{v}(\mathbf{q})=\partial_{\mathbf{q}} H_{\mathrm{GL}}(\mathbf{q})
$$

Within the Kubo linear response theory, the contribution of fluctuating CPs to the DC conductivity (and transconductivity $\left.\sigma_{\alpha \beta}^{\mathrm{D}}=-\sigma_{\alpha \beta}\right)$ tensor $\sigma_{\alpha \beta}=e^{2} \operatorname{Im}\left[\chi_{\alpha \beta}^{\mathrm{R}}(\omega)\right] / \omega$ is related to the imaginary part of the retarded velocity-velocity correlation function $\chi_{\alpha \beta}^{\mathrm{R}}(\omega)$. It can be obtained by the analytical continuation from the corresponding Matsubara correlation function $\chi_{\alpha \beta}\left(i \Omega_{n}\right)$, which is given by

$$
\chi_{\alpha \beta}\left(i \Omega_{n}\right)=T \sum_{p_{n} \mathbf{q}} \operatorname{Tr}\left[\hat{v}_{\alpha}(\mathbf{q}) \hat{L}\left(i p_{n}+i \Omega_{n}, \mathbf{q}\right) \hat{v}_{\beta}(\mathbf{q}) \hat{L}\left(i p_{n}, \mathbf{q}\right)\right]
$$

If we use the spectral representation of for the Cooper pair propagator, presented in Appendix A, the summation over Matsubara frequencies and the analytical continuation are straightforward and results in

$$
\sigma_{\alpha \beta}=\sum_{\mathbf{q} \gamma \gamma^{\prime}}\left(\operatorname{Re}\left[Q_{\alpha \beta}^{\gamma \gamma^{\prime}}(\mathbf{q})\right] J_{\operatorname{Re}}^{\gamma \gamma^{\prime}}(\mathbf{q})+\operatorname{Im}\left[Q_{x y}^{\gamma \gamma^{\prime}}(\mathbf{q})\right] J_{\operatorname{Im}}^{\gamma \gamma^{\prime}}(\mathbf{q})\right)
$$

Here $Q_{\alpha \beta}^{\gamma \gamma^{\prime}}$ represents the product of matrix elements for velocity operators and is given by

$$
Q_{\alpha \beta}^{\gamma \gamma^{\prime}}(\mathbf{q})=\left\langle\gamma \mathbf{q}\left|v_{\alpha}(\mathbf{q})\right| \gamma^{\prime} \mathbf{q}\right\rangle_{\mathrm{C} C}\left\langle\gamma^{\prime} \mathbf{q}\left|v_{\beta}(\mathbf{q})\right| \gamma \mathbf{q}\right\rangle_{\mathrm{C}}
$$

The factors $J_{\operatorname{Re}}^{\gamma \gamma^{\prime}}(p)$ and $J_{\operatorname{Im}}^{\gamma \gamma^{\prime}}(p)$ are given by

$$
J_{\mathrm{Re}}^{\gamma \gamma^{\prime}}=\int \frac{d \omega}{\pi} \operatorname{Im}\left[L_{\gamma}^{\mathrm{R}}\right] \operatorname{Im}\left[L_{\gamma^{\prime}}^{\mathrm{R}}\right]\left(-\frac{\partial n_{\mathrm{B}}(\omega)}{\partial \omega}\right), \quad J_{\mathrm{Im}}^{\gamma \gamma^{\prime}}=\int \frac{d \omega}{\pi}\left[\operatorname{Im}\left[L_{\gamma}^{\mathrm{R}}\right] \frac{\partial \operatorname{Re}\left[L_{\gamma^{\prime}}^{\mathrm{R}}\right]}{\partial \omega}-\operatorname{Im}\left[L_{\gamma^{\prime}}^{\mathrm{R}}\right] \frac{\partial \operatorname{Re}\left[L_{\gamma}^{\mathrm{R}}\right]}{\partial \omega}\right] .
$$

Here we use the compact notation $L_{\gamma}^{\mathrm{R}} \equiv L_{\gamma}^{\mathrm{R}}(\omega, \mathbf{q})$ for all Green functions inside the integrals. It should be noted that these relations are very general and do not rely on any specific frequency or momentum dependence of the Green function $L_{\gamma}^{\mathrm{R}}(\omega, \mathbf{q})$. For the Green functions in Eq. (52), which describe fluctuating CPs, we get

$$
\operatorname{Re}\left[L_{\gamma}^{\mathrm{R}}(\omega, \mathbf{q})\right]=\frac{\varepsilon_{\gamma \mathbf{q}}(p)+\omega \tau^{\prime \prime}}{\left(\omega \tau^{\prime}\right)^{2}+\left(\varepsilon_{\gamma \mathbf{q}}(p)+\omega \tau^{\prime \prime}\right)^{2}}, \quad \operatorname{Im}\left[L_{\gamma}^{\mathrm{R}}(\omega, \mathbf{p})\right]=\frac{\omega \tau^{\prime}}{\left(\omega \tau^{\prime}\right)^{2}+\left(\varepsilon_{\gamma \mathbf{q}}(p)+\omega \tau^{\prime \prime}\right)^{2}} .
$$

The integration in Eq. (59) can be performed and results in

$$
J_{\operatorname{Re}}^{\gamma \gamma^{\prime}}=\left(\frac{T}{\varepsilon_{\gamma \mathbf{q}}}+\frac{T}{\varepsilon_{\gamma^{\prime} \mathbf{q}}}\right) \frac{\tau^{\prime}|\tau|^{2}}{\left|\varepsilon_{\gamma \mathbf{q}} \tau+\varepsilon_{\gamma^{\prime} \mathbf{q}} \tau^{*}\right|^{2}}, \quad J_{\mathrm{Im}}^{\gamma \gamma^{\prime}}=\left(\frac{T}{\varepsilon_{\gamma \mathbf{q}}}-\frac{T}{E_{\gamma^{\prime}}}\right) \frac{\tau^{\prime \prime}|\tau|^{2}}{\left|\varepsilon_{\gamma \mathbf{q}} \tau+\varepsilon_{\gamma^{\prime} \mathbf{q}} \tau^{*}\right|^{2}} .
$$

It is instructive to consider intrachannel $\left(\gamma=\gamma^{\prime}\right)$ and intermode $\left(\gamma \neq \gamma^{\prime}\right)$ contributions to the conductivity tensor separately. The factor $J_{\operatorname{Im}}^{\gamma \gamma^{\prime}}$ vanishes at $\gamma^{\prime}=\gamma$ and the intramode contribution can be written as

$$
\sigma_{\alpha \alpha}^{\mathrm{AL}}=\sum_{\gamma \mathbf{q}} \frac{\operatorname{Re}\left[Q_{\alpha \alpha}^{\gamma \gamma}(\mathbf{q})\right]}{2 \varepsilon_{\gamma \mathbf{q}}^{3}} \frac{T|\tau|^{2}}{\tau^{\prime}}
$$

Here we have taken into account that $Q_{\alpha \bar{\alpha}}^{\gamma \gamma}=0$ and intramode transitions contribute only to the longitudinal conductivity. As it is discussed in the main part of paper, $\sigma_{\alpha \alpha}^{\mathrm{AL}}$ represents a sum of two independent terms describing the conventional Aslamazov-Larkin effect for each of two competing channels. It is instructive to separate the intermode contribution into two terms as follows

$$
\begin{aligned}
\sigma_{\alpha \bar{\alpha}}^{\Omega} & =\sum_{\gamma \mathbf{q}} \frac{\operatorname{Im}\left[Q_{\alpha \bar{\alpha}}^{\gamma \bar{\gamma}}\right] \tau^{\prime \prime}|\tau|^{2}}{\left|\varepsilon_{\gamma \mathbf{q}} \tau+\varepsilon_{\bar{\gamma} \mathbf{q}} \tau^{*}\right|^{2}}\left(\frac{T}{\varepsilon_{\gamma \mathbf{q}}}-\frac{T}{\varepsilon_{\bar{\gamma} \mathbf{q}}}\right)=\sum_{\gamma \mathbf{q}} \frac{2 \operatorname{Im}\left[Q_{\alpha \bar{\alpha}}^{\gamma}\right] \tau^{\prime \prime}|\tau|^{2}}{\left|\varepsilon_{\gamma_{\mathbf{q}}} \tau+\varepsilon_{\bar{\gamma} \mathbf{q}} \tau^{*}\right|^{2}} \cdot \frac{T}{\varepsilon_{\gamma \mathbf{q}}}, \\
\sigma_{\alpha \beta}^{\mathrm{G}} & =\sum_{\gamma \mathbf{q}} \frac{\operatorname{Re}\left[Q_{\alpha \beta}^{\gamma \bar{\gamma}}\right] \tau^{\prime}|\tau|^{2}}{\left|\varepsilon_{\gamma \mathbf{q}} \tau+\varepsilon_{\bar{\gamma} \mathbf{q}} \tau^{*}\right|^{2}}\left(\frac{T}{\varepsilon_{\gamma \mathbf{q}}}+\frac{T}{\varepsilon_{\bar{\gamma} \mathbf{q}}}\right)=\sum_{\gamma \mathbf{q}} \frac{2 \operatorname{Re}\left[Q_{\alpha \beta}^{\gamma / \bar{\gamma}}\right] \tau^{\prime}|\tau|^{2}}{\left|\varepsilon_{\gamma \mathbf{q}} \tau+\varepsilon_{\bar{\gamma} \mathbf{q}} \tau^{*}\right|^{2}} \cdot \frac{T}{\varepsilon_{\gamma \mathbf{q}}} .
\end{aligned}
$$

Here we have taken into account that $\operatorname{Im}\left[Q_{\alpha \alpha}^{\gamma \bar{\gamma}}\right]=0$. These terms represent anomalous Aslamazov-Larkin contributions to the conductivity. In Appendix $\mathrm{C}$ we demonstrate that they are intricately related with the nonzero Berry curvature $\left(\Omega_{\gamma \mathbf{q}}\right)$ and quantum metric $\left(\hat{G}_{\gamma \mathbf{q}}\right)$ for GL Hamiltonian $H_{\mathrm{GL}}$. 


\section{B2. Temperature dependence of the conductivity tensor}

This subsection presents derivation of conventional and anomalous Aslamazov-Larkin contributions to conductivity tensor. The explicit expression for the velocity operator $\mathbf{v}(\mathbf{q})$ introduced in Eq. (55) are

$$
v_{x}(\mathbf{q})=\left(\begin{array}{cc}
2 \xi \mathbf{q}_{x} & -\xi_{\star} \\
-\xi_{\star} & 2 l \mathbf{q}_{x}
\end{array}\right), \quad v_{y}(\mathbf{q})=\left(\begin{array}{cc}
2 \xi \mathbf{q}_{y} & i \xi_{\star} \\
-i \xi_{\star} & 2 l \mathbf{q}_{y}
\end{array}\right) .
$$

As a result, the product of matrix elements for velocity operators $Q_{\alpha \beta}^{\gamma \gamma^{\prime}}(\mathbf{q})$ averaged over the direction of momentum $\mathbf{q}$ is given by

$$
\begin{array}{lr}
\left\langle Q_{x x}^{\gamma \gamma}\right\rangle_{\phi_{\mathbf{q}}}=2 \xi^{4} \mathbf{q}^{2}+\frac{\xi_{\star}^{4} \mathbf{q}^{2}}{2 d_{\mathbf{q}}} \approx 2 \xi^{4} \mathbf{q}^{2}, & \left\langle Q_{x y}^{\gamma \gamma}\right\rangle_{\phi_{\mathbf{q}}}=0, \\
\left\langle Q_{x x}^{\gamma \bar{\gamma}}\right\rangle_{\phi_{\mathbf{q}}}=\frac{\xi_{\star}^{2}}{2}\left(1+\frac{\varepsilon_{z}^{2}}{d_{\mathbf{q}}^{2}}\right) \approx \xi_{\star}^{2}, & \left\langle Q_{x y}^{\gamma \bar{\gamma}}\right\rangle_{\phi_{\mathbf{q}}}=\zeta \xi_{\star}^{2} \frac{\varepsilon_{z}}{d_{\mathbf{q}}} \approx \zeta \xi_{\star}^{2} \operatorname{sign}\left[\varepsilon_{z}\right] .
\end{array}
$$

Here we have also performed the expansion in the ratio of spatial scales, $\xi_{\star} / \xi \ll 1$. Besides, in the leading order in $\xi_{\star} / \xi$ the effect of the effective pseudospin-orbit interactions at the dispersion of fluctuating CPs can be neglected. As a result, the dispersion relations are approximated as $\varepsilon_{\mathrm{A}(\mathrm{B})}+\xi^{2} q^{2}$. If we also expand in the ratio of time scales $\tau^{\prime \prime} / \tau^{\prime} \ll 1$, the expressions for the conductivity tensor Eqs. (62), (63), and (64) can be presented as

$$
\sigma_{x x}^{\mathrm{AL}}=\frac{e^{2}}{2 \pi \hbar} \frac{8 T \tau^{\prime}}{\pi} F_{\mathrm{AL}}, \quad \sigma_{y x}^{\Omega}=\zeta \frac{e^{2}}{2 \pi \hbar} \frac{8 T \tau^{\prime}}{\pi}\left(\frac{\xi_{\star}}{l}\right)^{2} \frac{\tau^{\prime \prime}}{\tau^{\prime}} F_{\Omega}, \quad \sigma_{x x}^{\mathrm{G}}=\frac{e^{2}}{2 \pi \hbar} \frac{8 T \tau^{\prime}}{\pi}\left(\frac{\xi_{\star}}{l}\right)^{2} F_{\mathrm{G}} .
$$

For the microscopically evaluated fluctuating CPs relaxation time $\tau^{\prime}$ given by Eq. (46), the second factor simplifies as $8 T \tau^{\prime} / \pi=1$. The temperature behavior of these terms is governed by dimensionless functions $F \equiv F\left(\varepsilon_{\mathrm{A}}, \varepsilon_{\mathrm{B}}\right)$. These functions depend only on $\varepsilon_{\mathrm{A}}=\ln \left[T / T_{\mathrm{A}}^{0}\right]$ and $\varepsilon_{\mathrm{B}}=\ln \left[T / T_{\mathrm{B}}^{0}\right]$ and are given by

$$
\begin{array}{r}
F_{\mathrm{L}}^{\mathrm{AL}}=\frac{\pi}{8} \int_{0}^{\infty} \frac{d\left(\xi^{2} q^{2}\right)}{2}\left[\frac{\xi^{2} q^{2}}{\left(\varepsilon_{\mathrm{A}}+\xi^{2} q^{2}\right)^{3}}+\frac{\xi^{2} q^{2}}{\left(\varepsilon_{\mathrm{B}}+\xi^{2} q^{2}\right)^{3}}\right]=\frac{\pi}{32}\left(\frac{1}{\varepsilon_{\mathrm{A}}}+\frac{1}{\varepsilon_{\mathrm{B}}}\right), \\
F_{\mathrm{G}}=\frac{\pi}{8} \int_{0}^{\infty} \frac{d\left(\xi^{2} q^{2}\right)}{2} \frac{1}{\left(\varepsilon_{\mathrm{s}}+\xi^{2} q^{2}\right)\left(\varepsilon_{\mathrm{A}}+\xi^{2} q^{2}\right)\left(\varepsilon_{\mathrm{B}}+\xi^{2} q^{2}\right)}=\frac{\pi}{8\left(\varepsilon_{\mathrm{A}}-\varepsilon_{\mathrm{B}}\right)^{2}} \ln \left[\frac{\left(\varepsilon_{\mathrm{A}}+\varepsilon_{\mathrm{A}}\right)^{2}}{4 \varepsilon_{\mathrm{A}} \varepsilon_{\mathrm{B}}}\right], \\
F_{\Omega}=\frac{\pi}{8} \int_{0}^{\infty} \frac{d\left(\xi^{2} q^{2}\right)}{2} \frac{\varepsilon_{z}}{\left(\varepsilon_{\mathrm{s}}+\xi^{2} q^{2}\right)^{2}\left(\varepsilon_{\mathrm{A}}+\xi^{2} q^{2}\right)\left(\varepsilon_{\mathrm{B}}+\xi^{2} q^{2}\right)}=\frac{\pi}{8\left(\varepsilon_{\mathrm{A}}-\varepsilon_{\mathrm{B}}\right)}\left\{\frac{\ln \left[\varepsilon_{\mathrm{A}} / \varepsilon_{\mathrm{B}}\right]}{\varepsilon_{\mathrm{A}}-\varepsilon_{\mathrm{B}}}-\frac{2}{\varepsilon_{\mathrm{A}}+\varepsilon_{\mathrm{B}}}\right\} .
\end{array}
$$

As a result, we recover the expressions Eq. (26), which are presented in the main text of the paper.

\section{APPENDIX C. KINETIC EQUATION FOR FLUCTUATING COOPER PAIRS}

\section{C1. Time-dependent Ginzburg-Landau equation}

This Appendix is devoted to the time-dependent Ginzburg-Landau (TDGL) equation. It governs the dissipative dynamics of the bosonic field $\Delta \equiv\left\{\Delta_{\tau \mathbf{r}}^{\mathrm{A}}, \Delta_{\tau \mathbf{r}}^{\mathrm{B}}\right\}$ for fluctuating CPs and is given by

$$
\tau^{*}\left(\frac{\partial \Delta_{t \mathbf{r}}}{\partial t}+i e \phi_{t \mathbf{r}}^{\mathrm{eh}} \Delta\right)=-\hat{H}_{\mathrm{GL}}(\mathbf{q}) \Delta+\eta_{t \mathbf{r}}, \quad\left\langle\eta_{t \mathbf{r}} \eta_{t^{\prime} \mathbf{r}^{\prime}}^{\dagger}\right\rangle=\hat{N} \delta_{t t^{\prime}} \delta_{r \mathbf{r}^{\prime}}, \quad \hat{N}=2 T \tau^{\prime} \hat{1}
$$

Here we take into account that components of fluctuating CPs have opposite charges and are spatially separated. For these reasons, coupling with electric potentials in both layers $\left(\phi_{t \mathbf{r}}^{\mathrm{e}}\right.$ and $\left.\phi_{t \mathbf{r}}^{\mathrm{h}}\right)$ can be introduced via the Peierls substitution $\partial_{t} \rightarrow \partial_{t}+i e \phi_{t \mathbf{r}}^{\mathrm{eh}}$ with $\phi_{t \mathbf{r}}^{\mathrm{eh}}=\phi_{t \mathbf{r}}^{\mathrm{e}}-\phi_{t \mathbf{r}}^{\mathrm{h}}$. The external complex field $\eta_{t \mathbf{r}}=\left\{\eta_{t \mathbf{r}}^{\mathrm{A}}, \eta_{t \mathbf{r}}^{\mathrm{B}}\right\}$ is the Langevin noise. Its presence is dictated by the fluctuation-dissipation theorem, and the correlation function $\left\langle\eta_{t \mathbf{r}} \eta_{t^{\prime} \mathbf{r}^{\prime}}^{\dagger}\right\rangle$, which is free of temporal and spatial correlations (white noise), is proportional to the relaxation rate $\tau^{\prime}$ but does not depend on $\tau^{\prime \prime}$. In the considered weak-to-moderate Cooper pairing regime, their ratio is small $\tau^{\prime \prime} / \tau^{\prime} \ll 1$; hence, the dissipation of fluctuating CPs is an essential component in their dynamics. These CPs cannot be interpreted as bosonic quasiparticles, but are instead overdamped bosonic modes. 
The TDGL equation can be derived microscopically (e.g. with the help of the Keldysh diagrammatic technique), or introduced in a phenomenological way as we do here. If we ignore the coupling to electromagnetic field (that can be reintroduced by the Peierls substitution), the dynamics of fluctuating CPs in TDGL is governed by the inverse retarded propagator $\hat{L}^{\mathrm{R}}(\omega, \mathbf{q})$. In equilibrium the bosonic field describing fluctuating CPs is not zero, but is shaped by the noise as $\Delta_{\omega \mathbf{q}}=\hat{L}^{\mathrm{R}}(\omega, \mathbf{q}) \eta_{\omega \mathbf{q}}$ (and $\Delta_{\omega \mathbf{q}}^{\dagger}=\eta_{\omega \mathbf{q}}^{\dagger} \hat{L}^{\mathrm{A}}(\omega, \mathbf{q})$ with $\hat{L}^{\mathrm{A}}(\omega, \mathbf{q})$ is the advanced CP propagator). If we assume that the Langevin noise does not have any temporal and spatial correlations (white noise), the correlation $F_{\Delta}^{\mathrm{dyn}}(\mathbf{q})=\left\langle\Delta_{t \mathbf{q}} \Delta_{t \mathbf{q}}^{\dagger}\right\rangle$ can be presented as

$$
F_{\Delta}^{\mathrm{dyn}}(\mathbf{q})=\int \frac{d \omega}{2 \pi} \hat{L}^{\mathrm{R}}(\omega, \mathbf{q}) \hat{N} \hat{L}^{\mathrm{A}}(\omega, \mathbf{q}), \quad F_{\Delta}^{\mathrm{td}}(\mathbf{q})=\int_{-\infty}^{\infty} \frac{d \omega}{2 \pi i} \frac{T}{\nu_{0} \omega}\left[\hat{L}^{\mathrm{R}}(\omega, \mathbf{q})-\hat{L}^{\mathrm{A}}(\omega, \mathbf{q})\right]
$$

and is determined by the pseudospin structure of the noise correlation function $\hat{N}$. According to the general philosophy of the Langevin approach, $\hat{N}$ is chosen in a such way that the correlation function $F_{\Delta}^{\text {dyn }}(\mathbf{q})$ matches with the one $F_{\Delta}^{\mathrm{td}}(\mathbf{q})=\left\langle\Delta_{\tau \mathbf{q}} \Delta_{\tau \mathbf{q}}^{\dagger}\right\rangle$, which is evaluated via the thermodynamic averaging and is also presented in Eq. (70). If we use the spectral representation for CP propagators $\hat{L}^{\mathrm{R}(\mathrm{A})}(\omega, \mathbf{q})$ given by Eq. (52), the integration over frequency $\omega$ is straightforward and results in

$$
F_{\Delta}^{\mathrm{td}}(\mathbf{q})=\sum_{\gamma}|\gamma, \mathbf{q}\rangle_{\mathrm{C}}\langle\gamma, \mathbf{q}| \frac{T}{\varepsilon_{\gamma \mathbf{q}}}, \quad F_{\Delta}^{\mathrm{dyn}}(\mathbf{q})=\sum_{\gamma, \gamma^{\prime}} \frac{|\gamma, \mathbf{q}\rangle_{\mathrm{C} C}\left\langle\gamma, \mathbf{q}|\hat{N}| \gamma^{\prime}, \mathbf{q}\right\rangle_{\mathrm{C} C \mathrm{C}}\left\langle\gamma^{\prime}, \mathbf{q}\right|}{\tau^{\prime}\left(\varepsilon_{\gamma \mathbf{q}}+\varepsilon_{\gamma^{\prime} \mathbf{q}}\right)-i \tau^{\prime \prime}\left(\varepsilon_{\gamma \mathbf{q}}-\varepsilon_{\gamma^{\prime} \mathbf{q}}\right)}
$$

For the noise correlation function given by the right-hand side expression in Eq. (69), $F_{\Delta}^{\text {td }}(\mathbf{q})$ and $F_{\Delta}^{\text {dyn }}(\mathbf{q})$ match each other. As a result, the description of fluctuating CPs within the quantum field theory approach and within the TDGL framework are consistent with each other.

\section{C2. Transformation of the TDGL equation into the kinetic equation}

This subsection presents derivation of the kinetic equation for fluctuating CPs. First, it is instructive to introduce a density matrix for fluctuating CPs as $\rho_{\mathbf{r r}^{\prime} t}=\Delta_{\mathbf{r} t} \Delta_{\mathbf{r}^{\prime} t}^{\dagger}$. The density matrix satisfies the equation

$$
\frac{\partial \rho_{\mathbf{r r} t}}{\partial t}=-i e\left(\phi_{\mathbf{r} t}^{\mathrm{eh}}-\phi_{\mathbf{r}^{\prime} t}^{\mathrm{eh}}\right) \rho_{\mathbf{r r}^{\prime} t}-\frac{\hat{H}_{\mathrm{GL}} \rho_{\mathbf{r r}^{\prime} t}}{\tau}-\frac{\rho_{\mathbf{r r}^{\prime} t} \hat{H}_{\mathrm{GL}}}{\tau^{*}}+\frac{\eta_{\mathbf{r} t} \Delta_{\mathbf{r}^{\prime} t}^{\dagger}}{\tau}+\frac{\Delta_{\mathbf{r} t} \eta_{\mathbf{r}^{\prime} t}^{\dagger}}{\tau^{*}},
$$

which can be interpreted as a generalization of the Liouville-von Neumann equation.

Second, we introduce a distribution function $\hat{n}_{\mathbf{R q} t}$ for fluctuating CPs. This function is obtained from the density matrix by the Wigner transformation and the unitary rotation to the eigenmode basis as follows:

$$
\hat{n}_{\mathbf{R q} t}=U_{\mathbf{q}}^{\dagger} \hat{n}_{\mathbf{R q} t}^{\prime} U_{\mathbf{q}} . \quad \hat{n}_{\mathbf{R q} t}^{\prime}=\int d \mathbf{r} e^{-i \mathbf{q r}} \hat{\rho}_{\mathbf{R}+\frac{\mathbf{r}}{2}, \mathbf{R}-\frac{\mathbf{r}}{2}, t}=\int \frac{d \mathbf{p}}{(2 \pi)^{2}} e^{i \mathbf{p R}} \hat{\rho}_{\mathbf{q}+\frac{\mathbf{p}}{2}, \mathbf{q}-\frac{\mathbf{p}}{2}, t} .
$$

Here, $U_{\mathbf{q}}$ is the unitary matrix that diagonalizes the GL Hamiltonian $\hat{H}_{\mathrm{GL}}$ as $U_{\mathbf{q}}^{\dagger} \hat{H}_{\mathrm{GL}} U_{\mathbf{q}}=\operatorname{diag}\left\{\varepsilon_{+, \mathbf{q}}, \varepsilon_{-, \mathbf{q}}\right\}$ and is given by Eq. (53). Diagonal elements of the rotated distribution matrix $\left(n_{++} \equiv n_{+}\right.$and $\left.n_{--}=n_{-}\right)$can be interpreted as distribution functions for eigenmodes in phase space $(\mathbf{R}, \mathbf{q})$. The off-diagonal elements $\left(n_{+-}\right.$and $\left.n_{-+}\right)$ are responsible for intermode coherence. For noise-induced equilibrium fluctuating CPs, $\Delta_{\omega \mathbf{q}}^{0}=\hat{L}^{\mathrm{R}}(\omega, \mathbf{q}) \eta_{\omega \mathbf{q}}$, the distribution function $\hat{n}_{\mathbf{q}}^{0}$ is position- and time-independent, as given below:

$$
\hat{n}_{\mathbf{q}}^{0}=\left(\begin{array}{cc}
n_{+, \mathbf{q}}^{0} & 0 \\
0 & n_{-, \mathbf{q}}^{0}
\end{array}\right) .
$$

At thermal equilibrium, there is no intermode coherence, and the diagonal terms are given by the classical distribution function $n_{\gamma \mathbf{q}}^{0}=T / \varepsilon_{\gamma \mathbf{q}}$, which confirms the interpretation outlined above for $\hat{n}_{\mathbf{R q} t}$.

Third, we will follow Ref. [84] and perform a gradient expansion of the equation for the matrix distribution function $\hat{n}_{\mathbf{R q} t}$. For a start, we apply the Wigner transformation to Eq. (72) and get the following equation for $\hat{n}_{\mathbf{R q} t}^{\prime}$

$$
\partial_{t} \hat{n}_{\mathbf{R q} t}^{\prime}=T_{1}+T_{2}+T_{3}
$$


The right-hand side is divided into three terms, which need to be dealt with separately. The term $T_{1}$ appears due to the coupling of fluctuating CPs with external electric field and within leading order of the gradient expression simplifies as

$$
T^{1}=-i e \int d \mathbf{r} e^{-i \mathbf{q r}}\left(\phi_{\mathbf{R}+\mathbf{r} / 2, t}^{\mathrm{eh}}-\phi_{\mathbf{R}+\mathbf{r} / 2, t}^{\mathrm{eh}}\right) \hat{\rho}_{\mathbf{R}+\frac{r}{2}, \mathbf{R}-\frac{r}{2}, t} \approx-e \mathbf{E}_{\mathbf{R} t}^{\mathrm{eh}} \partial_{\mathbf{q}} \hat{n}_{\mathbf{R q} t}^{\prime} .
$$

Here $E_{\mathbf{R} t}^{\mathrm{eh}}=-\nabla \phi_{\mathbf{R} t}^{\mathrm{eh}}$ is an electric field difference between layers. The term $T_{2}$ describes the dynamics of fluctuating CPs as

$$
T_{2}=-\frac{\hat{H}_{\mathrm{GL}} \otimes \hat{n}_{\mathbf{R q} t}^{\prime}}{\tau}-\frac{\hat{n}_{\mathbf{R q} t}^{\prime} \otimes \hat{H}_{\mathrm{GL}}}{\tau^{*}} \approx-\frac{\hat{H}_{\mathrm{GL}}(\mathbf{q}) \hat{n}}{\tau}-\frac{\hat{n} \hat{H}_{\mathrm{GL}}(\mathbf{q})}{\tau^{*}}+\frac{i \hbar}{2}\left(\frac{\partial_{\mathbf{q}} \hat{H}_{\mathrm{GL}} \partial_{\mathbf{R}} \hat{n}_{\mathbf{R q} t}^{\prime}}{\tau}-\frac{\partial_{\mathbf{R}} \hat{n}_{\mathbf{R q} t}^{\prime} \partial_{\mathbf{q}} \hat{H}_{\mathrm{GL}}}{\tau^{*}}\right) .
$$

Here $\otimes$ is the Moyal product, which in the lowest order of the gradient expansion simplifies as

$$
\otimes=\exp \left(\frac{i \hbar}{2}\left[\overleftarrow{\partial}_{\mathbf{R}} \vec{\partial}_{\mathbf{q}}-\overleftarrow{\partial}_{\mathbf{q}} \vec{\partial}_{\mathbf{R}}\right]\right) \approx 1+\frac{i \hbar}{2}\left[\overleftarrow{\partial}_{\mathbf{R}} \vec{\partial}_{\mathbf{q}}-\overleftarrow{\partial}_{\mathbf{q}} \vec{\partial}_{\mathbf{R}}\right]
$$

The term $T_{3}$ describes the effect of the Langevin noise and is of the first order in it. That is why we can approximate the order parameter by its value in equilibrium $\Delta_{\omega \mathbf{q}}^{0}=\hat{L}^{\mathrm{R}}(\omega, \mathbf{q}) \eta_{\omega \mathbf{q}}$ that allows to simplify $T_{3}$ as follows

$$
T_{3}=\int \frac{d \omega}{2 \pi}\left(\frac{\hat{N} \hat{L}^{\mathrm{A}}(\omega, \mathbf{q})}{\tau}+\frac{\hat{L}^{\mathrm{A}}(\omega, \mathbf{q}) \hat{N}}{\tau}\right)=\frac{2 T \tau^{\prime}}{|\tau|^{2}} \hat{1} .
$$

Here we have used the spectral decomposition for the Cooper pair propagator, Eq. (52). If we combine all three terms $T_{1}, T_{2}$ and $T_{3}$ together we get a closed-form equation for the distribution function $\hat{n}$ as

$$
\partial_{t} \hat{n}_{\mathbf{R q} t}^{\prime}=-e \mathbf{E}_{\mathbf{R} t}^{\mathrm{eh}} \partial_{\mathbf{q}} \hat{n}_{\mathbf{R q} t}^{\prime}-\frac{\hat{H}_{\mathrm{GL}}(\mathbf{q}) \hat{n}_{\mathbf{R q} t}^{\prime}}{\tau}-\frac{\hat{n}_{\mathbf{R q} t}^{\prime} \hat{H}_{\mathrm{GL}}(\mathbf{q})}{\tau^{*}}+\frac{i \hbar}{2}\left(\frac{\partial_{\mathbf{q}} \hat{H}_{\mathrm{GL}} \partial_{\mathbf{R}} \hat{n}_{\mathbf{R} \mathbf{} t}^{\prime}}{\tau}-\frac{\partial_{\mathbf{R}} \hat{n}_{\mathbf{R q} t}^{\prime} \partial_{\mathbf{q}} \hat{H}_{\mathrm{GL}}}{\tau^{*}}\right)+\frac{2 T \tau^{\prime}}{|\tau|^{2} \nu_{0}} \hat{1} .
$$

Now we are ready to perform the rotation $\hat{n}=U_{\mathbf{q}}^{\dagger} \hat{n}_{\mathbf{R} \mathbf{t}}^{\prime} U_{\mathbf{q}}$ to the eigenmode basis. Here, $U_{\mathbf{q}}$ is the unitary matrix that diagonalizes the GL Hamiltonian $\hat{H}_{\mathrm{GL}}$ as $U_{\mathbf{q}}^{\dagger} \hat{H}_{\mathrm{GL}} U_{\mathbf{q}}=\operatorname{diag}\left\{\varepsilon_{+, \mathbf{q}}, \varepsilon_{-, \mathbf{q}}\right\}$. The rotation of Eq. (79) results in

$$
\frac{\partial \hat{n}}{\partial t}=-e \mathbf{E}_{\mathbf{R} t}^{\mathrm{eh}} D_{\mathbf{q}} \hat{n}-\frac{\hat{E}_{\mathrm{GL}}(\mathbf{q}) \hat{n}}{\tau}-\frac{\hat{n} \hat{E}_{\mathrm{GL}}(\mathbf{q})}{\tau^{*}}+\frac{i \hbar}{2}\left(\frac{D_{\mathbf{q}} \hat{E}_{\mathrm{GL}} \partial_{\mathbf{R}} \hat{n}}{\tau}-\frac{\partial_{\mathbf{R}} \hat{n} D_{\mathbf{q}} \hat{E}_{\mathrm{GL}}}{\tau^{*}}\right)+\frac{2 T \tau^{\prime}}{|\tau|^{2} \nu_{0}} \hat{1} .
$$

Here $D_{\mathbf{q}} n=\partial_{\mathbf{q}}-i\left[\mathcal{A}_{\mathbf{q}}, n\right]$ is the covariant derivative. $\mathcal{A}_{\mathbf{q}}=i U_{\mathbf{q}}^{\dagger} \partial_{\mathbf{q}} U_{\mathbf{q}}$ is the generalized Berry connection, which has been introduced in the main part of the paper as Eq. (18). The equation for the diagonal elements of the distribution $\hat{n}_{\gamma}=$ can be presented as

$$
\partial_{t} n_{\gamma}+e \mathbf{E}^{\mathrm{eh}}\left[\partial_{\mathbf{q}} n_{\gamma}-i\left(\mathcal{A}_{\mathbf{q}}^{\gamma \bar{\gamma}} n_{\bar{\gamma} \gamma}-\mathcal{A}_{\mathbf{q}}^{\bar{\gamma} \gamma} n_{\gamma \bar{\gamma}}\right)\right]+\frac{\tau^{\prime \prime}}{|\tau|^{2}} \partial_{\mathbf{q}} \varepsilon_{\gamma \mathbf{q}} \partial_{\mathbf{R}} n_{\gamma}=-\frac{2 \varepsilon_{\gamma \mathbf{q}} \tau^{\prime}}{|\tau|^{2}}\left(n_{\gamma}-n_{\gamma}^{0}\right)
$$

The electric field difference, $\mathbf{E}_{t \mathbf{R}}^{\text {eh }}=-\partial_{\mathbf{R}} \phi_{t \mathbf{r}}^{\text {eh }}$ ), not only shifts the distribution functions $n_{\gamma}$, but also induces intermode coherence. The coupling with the off-diagonal components of the distribution function is governed by the generalized Berry connection matrix $\mathcal{A}_{\mathbf{q}}=i U_{\mathbf{q}}^{\dagger} \partial_{\mathbf{q}} U_{\mathbf{q}}$. The dissipative nature of fluctuating CPs dynamics is manifested in the presence of a relaxation term on the right-hand side of the kinetic equation. The equation for the off-diagonal elements is kept to the zeroth order of the gradient expansion (all gradient terms including $\partial_{t} \hat{n}_{\gamma \bar{\gamma}}$ are neglected). As a result, the intermode coherence $n_{\gamma \bar{\gamma}}$ adiabatically follows the disturbed distribution functions $n_{\gamma}$ as

$$
n_{\gamma \bar{\gamma}}=i \mathbf{A}_{\mathbf{q}}^{\gamma \bar{\gamma}} \partial_{\mathbf{R}} n_{+} \frac{\left(\varepsilon_{\gamma \mathbf{q}}-\varepsilon_{\bar{\gamma} \mathbf{q}}\right) \tau^{\prime \prime}}{\varepsilon_{\gamma \mathbf{q}} \tau^{*}+\varepsilon_{\bar{\gamma} \mathbf{q}} \tau}, \quad n_{\bar{\gamma} \gamma}=n_{\gamma \bar{\gamma}}^{*}
$$

Here we also have followed Ref. [84] and performed the approximation $\partial_{\mathbf{R}} n_{+} \approx \partial_{\mathbf{R}} n_{-}$, which decouples the kinetic equations for $n_{+}$and $n_{+}$. Besides, in this approximation predictions based on the resulting kinetic equations match with the ones based on the TDGL equation for fluctuating CPs and the linear response approach, as we discuss in the next subsection. If we combine expressions Eqs. (81) and (82), we obtain the kinetic equation, which is presented in the main part of the paper as Eq. (22). 


\section{C3. The conductivity tensor}

In this section we demonstrate that the expressions for the conductivity tensor derived with the help of the kinetic equation for fluctuating CPs match with results obtained in Appendix B with the help of the linear response theory. As we discuss in the main text, electric field difference $e \mathbf{E}^{\text {eh }}$ induces the three contributions to the current in both layers of the different physical origin

$$
\mathbf{J}^{\mathrm{e}(\mathrm{h})}= \pm \sum_{\mathbf{p} \gamma}\left[\partial_{\mathbf{q}} \varepsilon_{\gamma \mathbf{q}} n_{\gamma \mathbf{q}}^{1}+u_{\gamma \mathbf{q}}^{\Omega} n_{\gamma \mathbf{q}}^{0}+u_{\gamma \mathbf{q}}^{\mathrm{G}} n_{\gamma \mathbf{q}}^{0}\right], \quad n_{\gamma \mathbf{q}}^{0}=\frac{T}{\varepsilon_{\gamma \mathbf{q}}}, \quad n_{\gamma \mathbf{q}}^{1}=-\frac{|\tau|^{2} \mathbf{E}^{\mathrm{eh}} \partial_{\mathbf{q}} n_{\gamma \mathbf{q}}^{0}}{2 \tau^{\prime} \varepsilon_{\gamma \mathbf{q}}}
$$

The first term originates from the shift of the distribution function for fluctuating CPs and represents the conventional Aslamazov-Larkin effect. The corresponding contribution to the conductivity tensor is given by

$$
\sigma_{\alpha \beta}^{\mathrm{AL}}=\sum_{\gamma \mathbf{q}} \frac{\partial_{\mathbf{q}_{\alpha}} \varepsilon_{\gamma \mathbf{q}} \partial_{\mathbf{q}_{\beta}} \varepsilon_{\gamma \mathbf{q}}}{2 \varepsilon_{\gamma \mathbf{q}}^{3}} \frac{T|\tau|^{2}}{\tau^{\prime}}, \quad \partial_{\mathbf{q}_{\alpha}} \varepsilon_{\gamma \mathbf{q}} \partial_{\mathbf{q}_{\beta}} \varepsilon_{\gamma \mathbf{q}}=\operatorname{Re}\left[Q_{\alpha \beta}^{\gamma \gamma}(\mathbf{q})\right]
$$

Here we recall that $\left\langle\gamma \mathbf{q}\left|v_{\alpha}(\mathbf{q})\right| \gamma \mathbf{q}\right\rangle_{\mathrm{C}}=\partial_{\mathbf{q}_{\alpha}} \varepsilon_{\gamma \mathbf{q}}$. As a result, this expression matches with the Eq. (62) derived in Appendix B.

The second term originates from the anomalous velocity $u_{\gamma \mathbf{q}}^{\Omega}$, which is intricately related with the nonzero Berry curvature of fluctuating CPs. It results only in the Hall conductivity that is given by

$$
\sigma_{\alpha \beta}^{\Omega}=\epsilon_{\alpha \beta z} \sum_{\gamma \mathbf{q}} \Omega_{\gamma \mathbf{q}} \frac{\left(\varepsilon_{\gamma \mathbf{q}}-\varepsilon_{\bar{\gamma} \mathbf{q}}\right)^{2}\left|\tau^{\prime \prime} \tau\right|^{2}}{\left|\varepsilon_{\gamma \mathbf{q}} \tau^{*}+\varepsilon_{\bar{\gamma} \mathbf{q}} \tau\right|^{2}} \frac{T}{\varepsilon_{\gamma \mathbf{q}}}, \quad \Omega_{\gamma \mathbf{q}}=\frac{2 \operatorname{Im}\left[Q_{x y}^{\gamma \bar{\gamma}}\right]}{\left(\varepsilon_{\gamma \mathbf{q}}-\varepsilon_{\bar{\gamma} \mathbf{q}}\right)^{2}} .
$$

Here we have rewritten the Berry curvature $\Omega_{\gamma \mathbf{q}}$ in terms of the product of matrix elements for velocity operators. As a result, this expression matches with Eq. (63) derived in Appendix B.

The third term originates from the anomalous velocity $u_{\gamma \mathbf{q}}^{\mathrm{G}}$, which is intricately related with the nonzero quantum metric of fluctuating CPs. It results both in longitudinal and transverse elements of the conductivity tensor that are given by

$$
\sigma_{\alpha \beta}^{\mathrm{G}}=\sum_{\gamma \mathbf{q}} \hat{G}_{\gamma \mathbf{q}} \cdot \frac{\left(\varepsilon_{\gamma \mathbf{q}}^{2}-\varepsilon_{\bar{\gamma} \mathbf{q}}^{2}\right) \tau^{\prime}|\tau|^{2}}{\left|\varepsilon_{\gamma \mathbf{q}} \tau^{*}+\varepsilon_{\bar{\gamma} \mathbf{q}} \tau\right|^{2}} \frac{T}{\varepsilon_{\gamma \mathbf{q}}}, \quad \hat{G}_{\gamma \mathbf{q}}=-\frac{\operatorname{Re}\left[\hat{Q}^{\gamma \bar{\gamma}}\right]}{\left(\varepsilon_{\gamma \mathbf{q}}-\varepsilon_{\bar{\gamma} \mathbf{q}}\right)^{2}}
$$

Here we have rewritten the quantum metric $G_{\gamma \mathbf{q}}$ in terms of the product of velocity operators. If we use the following helpful identity

$$
\frac{\varepsilon_{+, \mathbf{q}}+\varepsilon_{-, \mathbf{q}}}{\varepsilon_{+, \mathbf{q}}-\varepsilon_{-, \mathbf{q}}}\left(\frac{T}{\varepsilon_{+, \mathbf{q}}}-\frac{T}{\varepsilon_{-, \mathbf{q}}}\right)=-\left(\frac{T}{\varepsilon_{+, \mathbf{q}}}+\frac{T}{\varepsilon_{-, \mathbf{q}}}\right)
$$

the expression Eq. (86) matches with the Eq. (64) derived in Appendix B. As a result, we conclude that the results obtained with the help of linear response approach and the kinetic equation are consistent with each other. 